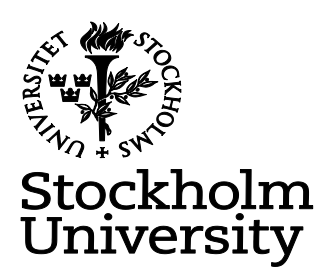

\title{
Long-term cognitive outcome of childhood traumatic brain injury
}

Catherine Aaro Jonsson 
(C)Catherine Aaro Jonsson, Stockholm 2010

ISBN 978-91-7447-054-3

Printed in Sweden by US-AB, Stockholm 2010

Distributor: Department of Psychology, Stockholm University

Cover photo: Elisabeth Aaro Östman 
To

Roger

Clara

Axel

and

Pontus 



\section{Abstract}

There is limited knowledge of cognitive outcome extending beyond 5 years after childhood traumatic brain injury, CTBI. The main objectives of this thesis were to investigate cognitive outcome at 6-14 years after CTBI, and to evaluate if advancements in the neurosurgical care, starting 1992, did influence long-term outcome and early epidemiology. An additional aim was to study the relationship between early brain injury parameters and early functional outcome. Study 1 evaluated cognitive progress during 14 years after CTBI, over three neuropsychological assessments in 8 patients with serious CTBI. Study 2 used patient records to investigate early epidemiology, received rehabilitation and medical follow up in two clinical cohorts, $n=82$ and $n=46$, treated neurosurgically for CTBI before and after 1992. An exploratory cluster analysis was applied to analyse the relation between early brain injury severity parameters and early functional outcome. In Study $\mathbf{3}$, participants in the two cohorts, $n=18$ and $n=23$, treated neurosurgically for CTBI before and after 1992, were subject to an extensive neuropsychological assessment, 13 and 6 years after injury, respectively. Assessment results of the two cohorts were compared with each other and with controls. Data were analysed with multivariate analyses of variance. Results and discussion. There were significant long-term cognitive deficits of similar magnitude and character in the two cohorts with CTBI, treated before and after the advancements in neurosurgical care. At 6-14 years after injury, long-term deficits in verbal intellectual and executive functions were found, and were discussed in terms of their late maturation and a decreased executive control over verbal memory-functions after CTBI. Visuospatial functions had a slightly better long-term recovery. The amount of rehabilitation received was equally low in both cohorts. The length of time spent in intensive care and the duration of care in the respirator may have a stronger relationship to early outcome than does a single measure of level of consciousness at admission. Main conclusions are that cognitive deficits are apparent at longterm follow up, 6-13 years after neurosurgically treated CTBI, even after advancements in the neurosurgical care in Sweden. Measures of verbal IQ, verbal memory and executive functions were especially low while visuospatial intellectual functions appear to have a better long-term recovery. 
Key-words: traumatic brain injury, childhood, adolescence, neurosurgical care, cognitive development, executive functions, memory, verbal functions, long-term outcome, recovery, rehabilitation, follow-up, cluster analysis. 


\section{List of publications}

Aaro Jonsson, C., Horneman, G., Emanuelson, I. (2004) Neuropsychological progress during 14 years after traumatic brain injury in childhood or adolescence. Brain Injury 18, 921-934.

Reprinted from Brain Injury, with permission from the publisher.

II Emanuelson, I., Aaro Jonsson, C., Rydenhag, B., Silander, H., Åkerman, A-K., Smedler, A-C. Traumatic brain injury in children treated at the Neurosurgical Intensive Care Unit at Sahlgrenska University Hospital during the years 1987-1991 and 1997-2001; an analysis of the process of care. Submitted.

Aaro Jonsson, C., Smedler, A-C., Leis Ljungmark, M., Emanuelson, I. (2009) Long-term cognitive outcome after neurosurgically treated childhood traumatic brain injury. Brain Injury $23,1008-1016$.

Reprinted from Brain Injury, with permission from the publisher. 


\section{Contents}

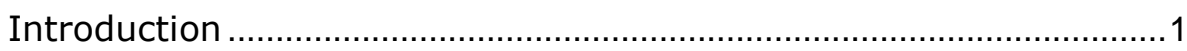

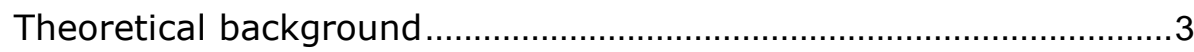

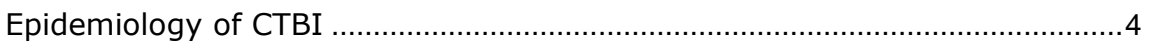

Injury characteristics .......................................................................

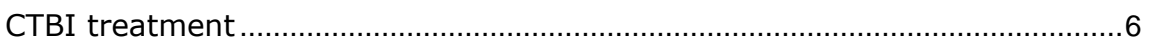

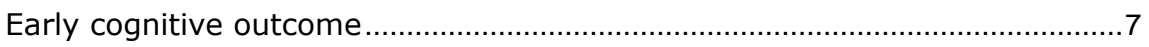

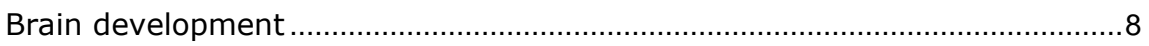

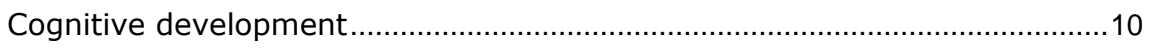

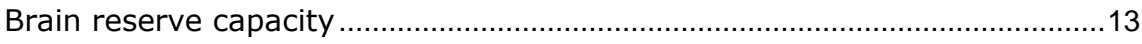

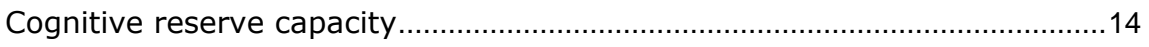

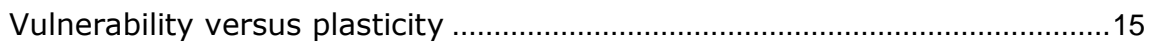

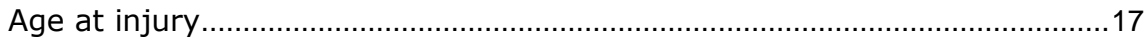

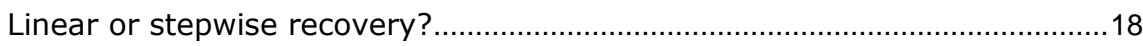

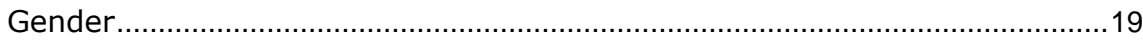

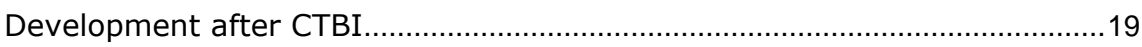

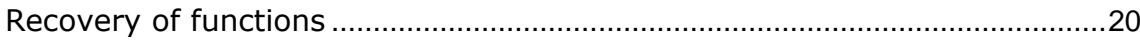

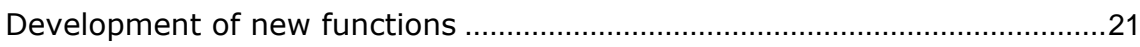

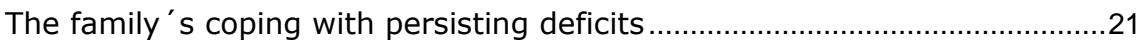

The child's coping with persisting deficits.......................................................23

The school's coping with persisting deficits.....................................................24

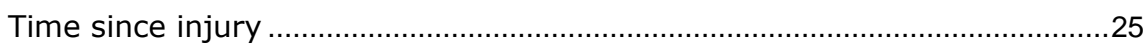

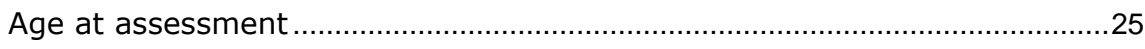

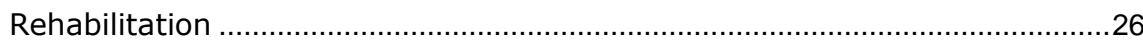

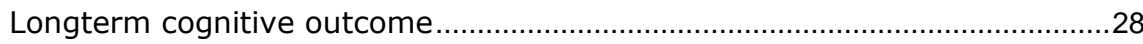

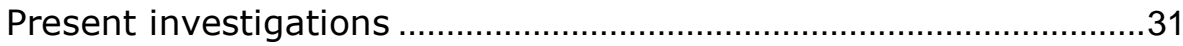

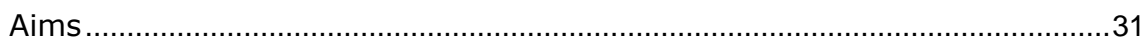

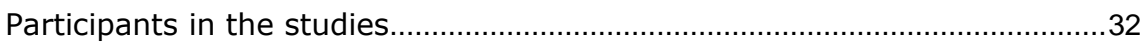

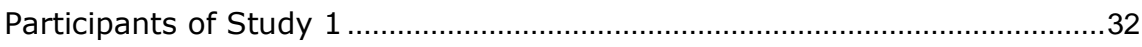

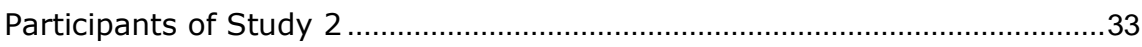

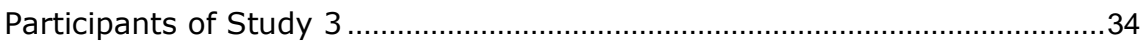

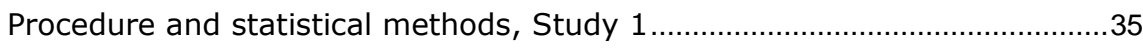

Procedure and statistical methods, Study 2..................................................36

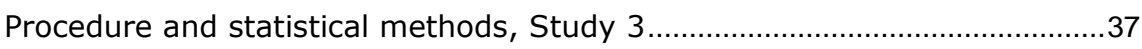

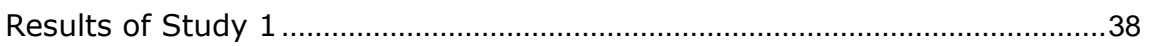




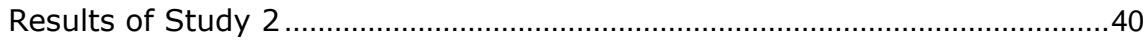

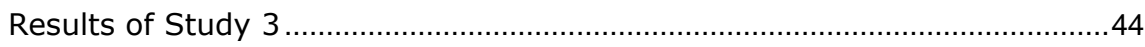

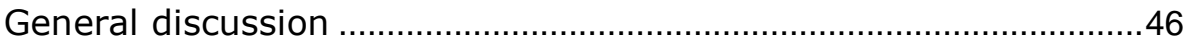

Verbal and visuospatial intellectual functions ..............................................4

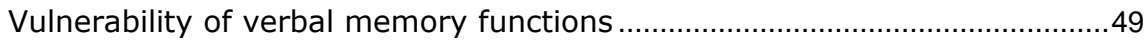

The connection between outcome and received rehabilitation .......................50

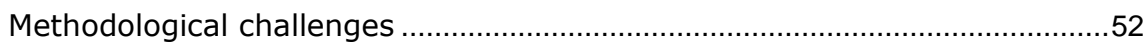

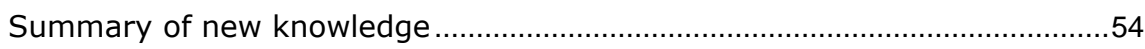

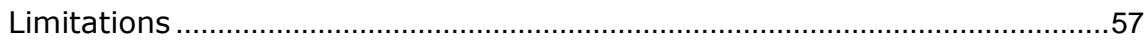

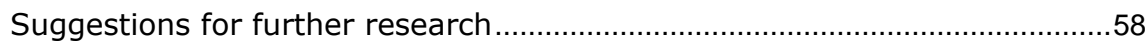

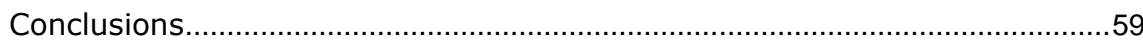

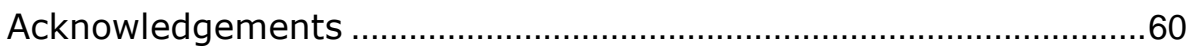

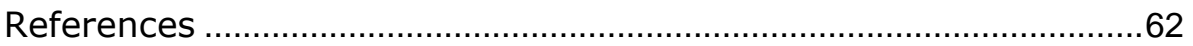





\section{Abbreviations}

\begin{tabular}{|c|c|}
\hline $\mathrm{BRC}$ & brain reserve capacity \\
\hline $\mathrm{CRC}$ & cognitive reserve capacity \\
\hline $\mathrm{ct}$ & Children's Category Test \\
\hline CTBI & childhood traumatic brain injury \\
\hline $\mathrm{d} 2$ tne & $\mathrm{d} 2$ Test of Attention, total numbers - errors \\
\hline FAS, or fas & Verbal Fluency Test (letters F, A, S) \\
\hline $\mathrm{ft} \mathrm{d}$ & Fingertapping Test, dominant hand \\
\hline $\mathrm{ft}$ nd & Fingertapping Test, non-dominant hand \\
\hline GCS & Glasgow Coma Scale \\
\hline GOS & Glasgow Outcome Scale \\
\hline ICD-10 & International Classification of Diseases, $10^{\text {th }}$ Revision \\
\hline ICD-9 & International Classification of Diseases, $9^{\text {th }}$ Revision \\
\hline PIQ & performance intelligence quotient \\
\hline rav ir & Rey Auditory Verbal Learning Test, immediate recall \\
\hline ravl & $\begin{array}{l}\text { Rey Auditory Verbal Learning Test, total correct } \\
\text { recalls }\end{array}$ \\
\hline ravrc & Rey Auditory Verbal Learning Test, recognition \\
\hline ravre & Rey Auditory Verbal Learning Test, delayed recall \\
\hline RLS & Reaction Level Scale \\
\hline ry $3 m$ & Rey Complex Figure Test, three min recall \\
\hline ryc & Rey Complex Figure Test, copy \\
\hline ryrc & Rey Complex Figure Test, recognition \\
\hline ryre & Rey Complex Figure Test, 30 min recall \\
\hline $\mathrm{sd}$ & standard deviation \\
\hline SPIQ & translated: a quick test of language based intelligence \\
\hline TBI & traumatic brain injury \\
\hline tmt-a & Trail Making Test - part A \\
\hline tmt-b & Trail Making Test - part B \\
\hline VIQ & verbal intelligence quotient \\
\hline WAIS & Wechsler Adult Intelligence Scale \\
\hline WAIS-R & Wechsler Adult Intelligence Scale - Revised \\
\hline wibm & Blockdesign WISC-III/WAIS-R \\
\hline wiof & Vocabulary WISC-III/WAIS-R \\
\hline WISC & Wechsler Intelligence Scale for Children \\
\hline WISC-III & Wechsler Intelligence Scale for Children, 3rd. ed \\
\hline
\end{tabular}





\section{Introduction}

A traumatic brain injury in childhood is one of those things that we wish did not happen. However, it does. Traumatic brain injuries, TBI, refer to the brain-injuries that are caused by an external force and come unexpectedly, interrupting a normal life. The two main causes of childhood traumatic brain injury, CTBI, are traffic accidents and falls. When an accident results in a brain injury, society need to be prepared to provide the initial emergency care. The child is taken care of and transported to a hospital and a decision is made whether it is a mild injury that mostly needs observation, or whether it is a more severe injury that needs intensive care. In the present thesis we have studied the children that acquire a brain injury of such severity that neurosurgical intensive care were needed. This group varies regarding the grade of severity. For example, the time spent in the intensive care differs a great deal between children with milder injuries and children with severe ones, from a few days up to over a month. Scientific advancements have resulted in therapeutic improvements and the survival rates for adult patients are higher today than one or two decades ago. We know that among adult TBI patients, the higher rate of survivors has not led to more patients in a vegetative state. We also know that the psychosocial problems involving work adjustment, marital conflicts, dependence and economy have been reported to increase amongst the adults affected by a TBI. This means that the group of adult patients leaving the neurosurgical intensive care after a traumatic brain injury is different from what it was 20 years ago. One of the main objectives of this thesis is to address the question of whether developments in neurosurgical intensive care have resulted in any changes in outcome for children who have suffered traumatic brain injury. The other main objective is to contribute to the investigation of the long-term cognitive outcome after a traumatic brain injury in childhood. From earlier research on children who have suffered a serious brain injury, we know that impairments in cognitive skills are common, and that cognitive and behavioural impairments sometimes become more visible over time when the earlier acquired capacity is insufficient to master daily activities. Cognitive problems can be hard to distinguish in daily life, and the character of the problems also changes over time and unfortunately often ends up as unmet or unrecognized needs. When it comes to cognitive outcome several years after a traumatic brain injury in childhood, our knowledge is limited. In order for society to be prepared to take care of the group of children that have remaining cognitive 
deficits after a traumatic brain injury, we need to know what the main cognitive features are. Which functions are vulnerable, and which functions might be more robust in a long-term perspective? Are the cognitive problems seen today of a different kind from what we saw 20 years ago, before the therapeutic improvements in acute care? The overall aim of this thesis is to contribute to the body of knowledge in this field. 


\section{Theoretical background}

The connection between childhood traumatic brain injury, CTBI, and functional outcome is complex, and to a large extent remains unexplained, even after grouping children into traditional classifications according to the severity of their injury (Taylor, 2006). Factors that have been shown to predict the functional outcome are: the severity of injury, location of injury, age at time of injury, time since injury (Anderson et al, 2005, 2006; Anderson \& Catroppa, 2006) and family factors (Anderson et al., 2006; Anderson \& Catroppa, 2006; Yeates et al, 2005) and the premorbid function of the child (Anderson et al, 2006; Yeates et al, 2005). In addition, gender has been reported to influence the early outcome of adults with TBI (Rogers \& Wagner, 2006) and cognitive functioning after CTBI (Donders \& Hoffman, 2002; Donders \& Woodward, 2003; Niemeier, Marwitz \& Lesher, 2007). The type of neurological intensive care provided has influenced the early outcome of adults with TBI (Eker, 2000) and later rehabilitation methods and techniques are known to influence the long-term cognitive outcome (Laatsch et al., 2007). In recent years, two other variables have been presented as moderators of the outcome after a CTBI (Dennis, Yeates, Taylor, Fletcher, 2007). They are the brain reserve capacity (BRC), meaning the passive capacity of the brain to function in a deficit free manner after a trauma, and the cognitive reserve capacity (CRC), the ability to optimize or maximize performance through differential recruitment of brain networks (Stern 2002). In this thesis, the focus is on the long-term cognitive outcome of CTBI. An overview by a model of the relation between CTBI and cognitive outcome might contribute to making the complex situation a bit more coherent. The model presented here is an extension of the developmental model of reserve capacity moderating functional outcome of CTBI presented by Dennis et al (2007).

The injury inflicted when a CTBI occurs and long-term cognitive outcome constitutes the main variables in the model. The mediating variables between the start of the morphological injury and the end point of cognitive outcome are brain reserve capacity and cognitive reserve capacity, which determine the functional plasticity, responsible for the recovery of injured functions and the development of new ones. The CTBI treatment moderators are the initial treatment and the long-term rehabilitation. The patient-related variables of age at injury, gender, time since injury, present age and ability to cope each influence, at different periods, the process between the injury and 
final long-term cognitive outcome. Each concept in the model is discussed in the following text.

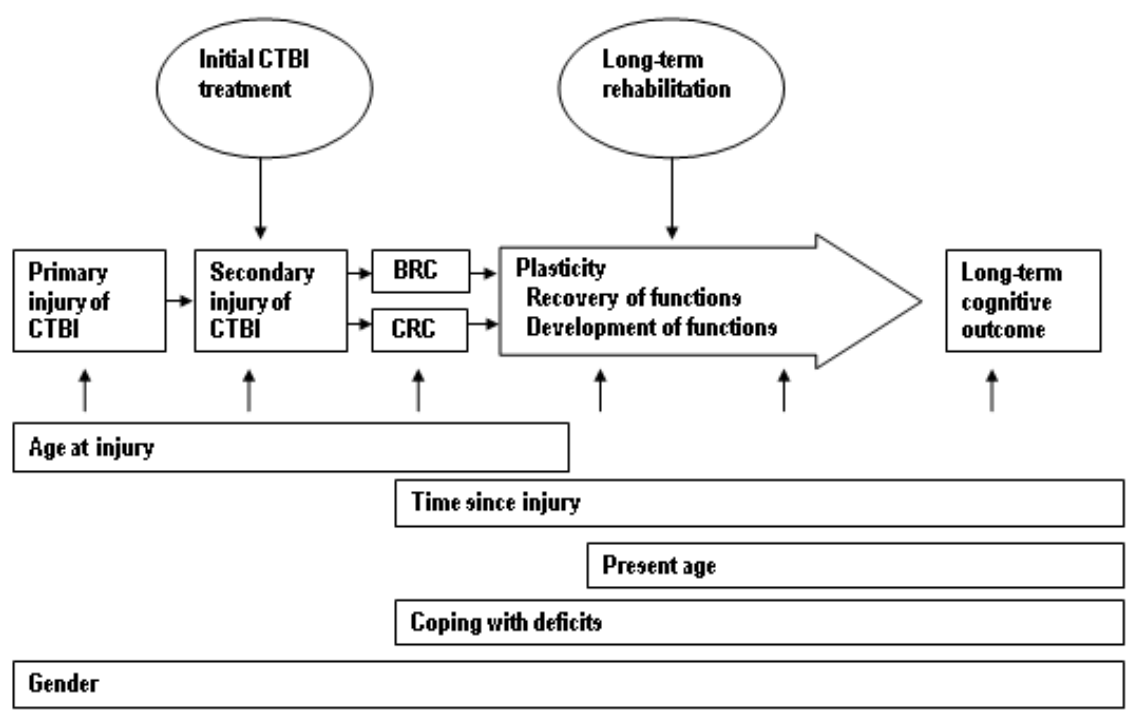

FIGURE 1. Influences on long-term cognitive outcome after a CTBI

\section{Epidemiology of CTBI}

Worldwide, injury is the main cause of death and disability in children (Sergui-Gomez \& MacKenzie, 2003). After the post-neonatal period, traumatic brain injury (TBI) are the most common type of acquired brain injury. A traumatic brain injury (TBI) is an injury to the central nervous system caused by an external force acquired after the neonatal period. The international annual incidence of TBI in children (CTBI) is 180/100 000 (Kraus, 1995), when all grades of severity of injury are included. Boys are more often than girls admitted to paediatric intensive care units for CTBI (Parslow, Morris, Tasker, Forsyth \& Hawley, 2005).

In the United States, 475000 children under 14 years of age sustain a TBI each year, with $90 \%$ of them being released immediately upon being seen in the emergency department (Keenan \& Bratton, 2006). Anderson \& Catroppa (2006) reported that epidemiology data from the United States suggest that 200 per 100000 children will experience TBI each year. Half of them will seek medical attention and according to a study by Emanuelson \& von 
Wendt (1997) 5-10\% of them will experience temporary and/or permanent neuropsychological impairment. The most common external causes of CTBI in a Swedish population of CTBI patients were traffic accidents, where the children were passengers or bicyclists. In the same population in the UK, pedestrian accidents were the most common causes of injury, and most often occurred in the late afternoon and early evening, with most admissions to intensive care occurring in the summer (Parslow et al., 2005). In a study of Australian children, those with mild TBI were more likely to have sustained injuries in falls, while severe injuries were most often caused by motor vehicle accidents; moderate injuries were more evenly distributed between falls and motor vehicle accidents (Anderson et al., 2001).

\section{Injury characteristics}

During and after a traumatic insult to the brain, the course of events is intense, complex and not completely understood; the ensuing injuries are, however, separated into primary and secondary injuries (Emanuelson, 1999). A primary injury arises from a mechanical external force. Secondary injuries, in contrast, occur minutes to days after the primary insult and are manifested by symptoms such as cerebral oedema and an elevation of intracranial pressure (Emanuelson, 1999). Secondary injuries are caused by the response of the brain to a primary injury, and, during the last few decades they have been implicated as a major contributor to worsened morbidity and mortality (Ylvisaker et al., 2005). Salorio et al (2008) suggested that an altered blood pressure and hypotension could be markers of secondary injury and may be used as predictors of the cognitive outcome 1 year post-injury.

A brain injury can be described as focal, diffuse or a combination of the two (Vik, Kvistad, , Skandsen \& Ingebrigtsen, 2006). A traumatic brain injury is different from that of a haemorrhage due to the force of the traumatic insult to the brain. Diffuse axonal injury is common after traffic injuries (Vik et al., 2006), and the consequence of non-penetrating CTBI is more often a diffuse injury (Levin, 2003). A diffuse injury results from sudden acceleration and deceleration and the simultaneous rotation of the freely moving head, and is further exacerbated by a secondary injury associated with ischemia, brain swelling and the release of excitatory neurotransmitters (Levin, 2003). Axonal damage often occurs hours or days after a primary injury. Vik et al. (2006) reported that recent studies have shown that secondary injuries most often occur as a result of oedema. The most common location of axonal injuries is in the connections between white and grey matter within the frontal and temporal lobes (Vik et al., 2006). Wilde et al. (2007) reported a symmetric decrease in the volumes of subcortical structures 1-10 years after a CTBI, lending support to the understanding that an injury is also global and diffuse rather than being of a solely focal nature. In the 
study, the hippocampus was revealed to be especially vulnerably to TBI, even after exclusion of children with hippocampal lesions, further supporting the global nature of TBI. Of focal CTBIs, the frontal region is most frequently involved (Mendelsohn et al., 1992).

Injury severity grades are usually defined by the level of consciousness after the injury. Clinically, they are most commonly measured by the Glasgow Coma Scale, GCS, which evaluates a patient's motor, verbal and eyeopening responses (Teasdale \& Jennett., 1974). A severe injury corresponds to a level below 8 , a moderate injury would have a score between eight and 12 and a mild injury corresponds to the levels 13-15 (Teasdale \& Jennett., 1974). The Swedish Reaction Level Scale, RLS, (Starmark, Stålhammar \& Holmgren, 1988) can assess overall responsiveness or consciousness level, without the use of verbal cues or eye-opening. That can be an advantage with young children, intubated patients or patients with swollen eyelids. For adults, agreement between the GCS and the RLS has been found to be good, with RLS functioning as well as the GCS for the critically injured (Walther, Jonasson \& Gill, 2003). In a study of adults and a small group of children, Johnstone et al. (1993) found that both scales function well in cases of severe and minor head injury, but he found that both have weaknesses when defining moderate head injuries. As one would expect, the more severe the grade assigned to a CTBI, the worse the anticipated outcome (Anderson et al., 2001). The description of outcome varies within the group classified as being severe from one country to another, but among the severely injured the following numbers are usually reported: a mortality rate of one third, with most deaths occurring before hospital admission, a good recovery for one third, and the remaining third of the patients will exhibit residual disability (Anderson \& Catroppa, 2006).

\section{CTBI treatment}

In the past decades, knowledge has emerged about the relationship between the secondary insults, and the morbidity and mortality of patients with CTBI, and thus many clinical trials have attempted to address secondary injuries to improve the long-term outcome (Ylvisaker et al., 2005). In Sweden, the "Lund Protocol" for the neurosurgical treatment of patients with severe TBIs aims at controlling intracranial pressure (Grande 1989, 2006; Asgeirsson, Grande \& Nordström, 1994). It was introduced in 1992 and evaluated on the adult population. A comparison with patients treated with an older neurosurgical method revealed that the mortality was markedly reduced, while the number of patients in a vegetative state remained on the same level. In contrast, psychosocial problems involving work adjustment, marital conflicts, dependence and financial problems were reported to increase. More than $40 \%$ of the group were reported to be dependent on rela- 
tives to cope with their daily life (Eker, Schalén, Asgeirsson, Grände, Ranstam, \& Nordström, 2000). When children with TBI were treated according to the Lund protocol, Wahlström, Oliviecrona, Koskinen, Rydenhag \& Naredi (2005) reported that $80 \%$ of them had a favourable outcome, indicated by a level of 4 or 5 on the Glasgow Outcome Scale (GOS). Moderate disability refers to level 4 on the scale and implies that a person assigned to this level of injury will be disabled, but independent, and therefore, for example, able to work in sheltered environments (Jennett \& Bond, 1975).

In the setting in which the initial treatment occurs, the first step in a potential rehabilitation process is taken with the decision made about whether a child could benefit from rehabilitation. This issue may be influenced by feelings from both medical professionals and family members that the child has been put through enough, having survived an accident and been subjected to many medical procedures to ensure his or her survival. Unfortunately, for many children with moderate and severe injuries, sequelae remain after the point at which medical stability is reached, and rehabilitation is needed to optimise recovery and the re-entry into everyday life (Beaulieu, 2002). Tepas et al. (2009) evaluated the relationship between a delay in the transition from acute care to rehabilitation, and found that comprehensive delays in the rehabilitation diminished the outcome in the severe group. In the moderate group, the delay was related to an increase in the duration of the rehabilitation. This result implies the need for a seamless transition during the early care to optimise the recovery during the window of opportunity that presents itself in the early stages following a CTBI. Beaulieu (2002) states that it is unwise to put one's faith in a recovery after a CTBI in the neural plasticity itself, remarking that "while neural plasticity offers the potential for reorganisation, it is the behavioural demands of the environment that allows the organism to take advantage of this potential and to maximise recovery" (p 393).

\section{Early cognitive outcome}

The early consequences of a CTBI are seen in a wide range of areas, including gross and fine motor problems, problems with speech like dysarthria, cognitive impairments and behavioural adjustment deficits (Anderson et al., 2001; Anderson \& Catroppa, 2006). In the initial phase after a CTBI, the injury-related cognitive characteristics differ markedly depending on the severity of the injury. Anderson et al. (2001b) reported that after a period of 6 months had elapsed since receiving a CTBI 50\% of those with severe injuries were found to have moderate to severe problems in multiple functional domains. In the moderate group, $40 \%$ had both physical and memory problems. 
Once a child has recovered sufficiently to have regained her general orientation, and has become goal directed and purposeful relative to her age expectations, she will generally return to his or her previous everyday life, at home and at school. Owing cognitive deficits, depending on the complexity of the tasks and the situations in which she is to be conducted, the child is likely to perform at different levels. Concrete situations requiring only minor cognitive or emotional demands can work out fine, however the child's behaviour or the efficiency of her information processing may deteriorate with an increase in the cognitive demands or psychosocial stress (Ylvisaker, 1998). Unfortunately, descriptions of the functions that are more resistant to CTBI are rare in the literature. However, one exception is the report that procedural memory, a form of implicit memory that is either perceptualmotor or cognitive and mediated through inferior and posterior regions, is less likely to be affected in children and adolescents with moderate-severe TBI (Ward, Shum, Wallace \& Boon, 2002).

Difficulties with attention is very common in the early stage after CTBI. In a follow-up study conducted 1 year after the injury, Kramer et al. (2008) a group with CTBI and a control group with orthopaedic injuries activated similar networks relevant to sustained attention processing, but the group with CTBI showed a neural over-activation in these areas. The results for the group with CTBI contrasted with the under-activation documented in studies of children with attention-deficit/hyperactivity disorder, commonly referred to as ADHD (Kramer et al., 2008). Levin et al. (2007) investigated changes in children's symptoms related to inattention and hyperactivity 2 years after CTBI. In a group that had been diagnosed as having ADHD prior to receiving their injury, symptoms related to hyperactive/impulsive behaviour and inattention predominated after the injury, while symptoms relating to a lack of attention were most common in the group where ADHD had only been diagnosed after the injury (Levin et al., 2007).

\section{Brain development}

The human nervous system begins to develop at 18 days gestation. About ten days later three major divisions of the brain are already discernible: the forebrain, that will later form the cerebral hemispheres, the limbic system, thalamus and hypothalamus; the midbrain forming part of the brainstem; and the hindbrain, hosting pons, medulla and cerebellum (Rosenzweig, Breedlove \& Watson, 2007). The production of nerve cells, the neurogenesis, takes place in the ventricular zone. The cells that will give rise to neurons either transform into nerve cells or into glial cells. The nerve cells formed at the ventricular level migrate along a particular kind of glial cell to their final destination in the emerging brain. Once in site, the process of celldifferentiation starts, in which the neuron matures to attain the specific ap- 
pearance and the function characteristic of that region. An extensive growth of axons, dendrites and the proliferation of synapses then takes place, thus starting up the communication between the cells (Rosenzweig et al., 2007). Around birth, there is a large overcapacity of cells and those that make adequate synapses remain, others die. Owing to this surplus in neurons, children have the capacity to adjust to different environments. Periods of cell death have been found to be followed by periods of synapse remodelling, where some synapses are lost, while others are formed. This is evident in the thinning of the grey matter of the cortex as pruning of dendrites and axons makes synaptic connections more efficient (Rosenzweig et al., 2007). Glial cells are essential for developing the communication between cells. In the process of myelinisation, sheaths are developed around the axons, greatly speeding up the rate at which axons conduct messages (Rosenzweig et al., 2007). Anderson (2007) has summarized a number of general rules for the myelination of the cerebral regions, suggesting that proximal pathways become myelinated before distal ones, sensory pathways before motor ones, projection areas before association areas, central areas before the poles, and that posterior zones become myelinated before anterior ones. However, the selection of which connections are to be myelinated is guided by an interaction between genes and experience. One example of the interaction is that synaptic stimulation influences which type of genes is activated. The impact of experience on neural development is best exemplified by the visual system, for which there appear to be sensitive periods during which lack of experience can exert long-lasting negative effects. In the visual cortex, these effects are most extensive during the early periods of synaptic development, and visual deprivation in this period results in a loss of dendritic spines and a reduction in the synapses. Animal studies have shown that several weeks of visual deprivation during a sensitive period results in blindness, and manipulation after the sensitive period will have little effect (Rosenzweig et al., 2007).

Magnetic resonance imaging (MRI) have revealed good options to study the normal structural brain development after birth in a safe way across agegroups. (Evans 2006, Casey et al 2000). In a study of Gogtay et al (2004) the thinning of grey matter was used as an indicator of brain maturation. They used MRI-scans every other year during 8 to 10 years in the study of the brain maturation of 13 healthy children, age 4-21. The result showed an overall increase of the grey matter thickening before puberty, with a following decrease thereafter. The maturation started in primary areas, associated with more basic functions of sensory-motor functions, and processing of vision, olfactory and taste. Temporal regions were the last to mature. The direction of the thinning of cortical grey matter had a back to front progression in the frontal lobe, reflecting a late maturation of the prefrontal lobe. In accordance with the view of cortical grey matter loss as a sign for maturation, increasing cognitive capacity is described to coincide with a gradual 
loss of synapses, and a strengthening of remaining synapses (Gogtay et al 2004). Lebel et al (2008) reported from a study of 202 persons between five and 30 years that most areas in the brain changed between ages of 5 and 30 . One of the exceptions was the fornix, a white matter connection involved in basic memory and emotion, appeared to be more mature than other regions during infancy. Most areas matured in adolescence while a few continued into the twenties with the fronto-temporal connections of cingulum and uncinate beeing two of the last to mature. Both studies give support for a nonlinear brain maturation (Lebel et al 2008, Gogtay et al 2004).

Children and adults appear to use the brain in different ways. Casey et al (2000) compared brain activation of children and adults on tasks of attention and inhibitory control. Brain activation was found in the similar frontal areas but the activation was two to three times higher for the children compared to the adults, and still the children had more difficulties in the task. Another study described in the same article, reported activation on similar cortical regions on spatial working memory tasks for school aged children and adults. Their performance reached the same level at the beginning of the task, but at the end of the scan session the children's performance had significantly deteriorated. Compared to the children, the performance of the adults continued to improve as a function of time on task (Casey et al 2000). These results are god examples of the notion that brain functions differ between children and adults, and that brain maturation can result in both higher efficacy and higher endurance.

\section{Cognitive development}

The brain development and the cognitive development mutually influence and are mutually dependent of each other (Casey et al 2000), a fact that is particularly noticeable after a CTBI . Ylvisaker (1998) summarised broad interrelated trends in the normal cognitive development, aiming at providing a developmental template linked to themes that are often influenced by a traumatic brain injury in childhood. The themes are beneath presented in italics.

A progression from the concrete to the abstract and hypothetical. In terms of cognitive development, the first 2 years in human life correspond to the sensory-motor stage of cognitive development described by Piaget (1982) in which the child explores the surroundings in a concrete way through sensory and motor abilities. Anyhow, a first sign of purposeful goal directed actions is seen already in infants (von Hofsten, 2004). When executing actions or observing the actions of others, infants fixate goals and sub-goals of the movements. When, for example, reaching for an object, the posture of the hand will adjust to the orientation of the object. Thus, the goal state is already represented when actions are planned (von Hofsten, 2004). The devel- 
opment of working memory is crucial to the ability of reflection, necessary for the later emerging abilities of hypothetical thinking. The first signs of working memory and the functionally close connected inhibitory control are seen between 7 and 8 months of life when infants can retrieve objects on a delayed response task when the delay was limited to 1-2 seconds (De Luca $\&$ Leventer, 2008). This is accompanied by early signs of progress in the frontal lobe seen between seven and 12 months (Bell \& Fox, 1994). Major gains in working memory are seen during the following years (De Luca \& Leventer, 2008). The executive functions of inhibitory control and sustained attention improve until the age of 5, and together with gains in working memory and strategy formation, they contribute to the maturation of the following new level of planning and goal-directed behaviour (De Luca \& Leventer, 2008).

In many societies, the age of 6 or 7 marks the beginning of a more structured education and period of socialisation. This coincides with the start of the most prominent progress in the development of attention control and performance speed (Anderson et al, 2001a). The earlier affective and sensory motor dependent memory functions change in favour of a more language and symbolic based memory (Piaget, 1982). The schoolchild reaches the stage of concrete operational cognition meaning that she has developed a new ability of reversible thinking with which she can imagine changes of situations she has experienced. The child will be able to think logically about a situation without having to try it in the real world (Piaget 1982). The development of executive functions is rapid during middle childhood and a developmental spurt of goal-setting skills occurs around 12 years of age (Anderson et al, 2001a). The further development of working memory will be attributable to increased processing efficiency of activation, inhibitory control and strategic functioning . In a study of the relation between working memory and inhibitory control, there was no correlation between functions of inhibitory control and working memory between six and 11 years of age, while the correlation was seen between 12 and 17 years, implying a stronger relation between these functions in adolescence (Roncadin, Pascual-Leone, Rich \& Dennis, 2007).

A progression from surface to depth, for example, a development of awareness from attending to the superficial into a comprehension of underlying causes (Ylvisaker, 1998). Owing to the increased memory capacity, around 18 months the child reaches the level of object permanence, meaning that the she now fully understands that an object still exists even when she cannot see it (Piaget, 1982) thus enabling the child to be aware of things that are not present at the surface. Around 6 to 7 years of age, in the stage of concrete operational cognition, the child will be able to imagine and understand how objects in the present surface can be changed by different possible actions (Piaget, 1982), therefore reaching a new understanding of that the surface can change, even though the if underlying conditions are the same. 
During adolescence, the theme of a comprehension of underlying causes is prominent. The capacity of attention and processing speed increases gradually throughout adolescence (Anderson et al., 2001a) and the cognitive development makes important advances, moving into the stage of formal operations (Piaget, 1982) in which a new ability for logical deductive cognition enables the adolescent to use a general principle to determine a specific outcome. The adolescent is not, as before, reliant on impressions from the outer world or earlier experiences to form new thoughts. This frees the mind from the experienced reality and often opens up an interest in philosophy, politics, religion, ideals or ethics, all to do with changing the outer world.

Growth in meta-cognition, e.g., progression to strategic thinking and problem-solving. Hanten (2004) summarises the metacognitive development of the child. Young children can understand that an instruction to remember requires a special effort, but up to the age around 7, they can't produce effective learning strategies. Not until the age of 8 , children implement longer study-times when they are supposed to remember things for a long time or when they are supposed to remember more difficult information. For the schoolchild, an increasing inhibitory control contributes to a new independence towards adults. When younger, the child may have difficulty resisting the temptation to satisfy her immediate needs even if satisfying them is in conflict with external rules. At these ages, children can also independently assess social conditions that lead to social emotions, such as shame, guilt and pride (Havnesköld \& Risholm Mothander, 2009).

Progression from ego-centric to non-egocentric thinking and action.. Mentalising, that is, the ability to discern the mental state of others is an prerequisite for the participating in a socially shared and predictable world. Mentalising, or it's precursors might be seen as early as at the end of the first year of life in the infant's ability to understand teasing (Frith \& Frith, 2007). From the age of 18 months, it is seen in the infant's understanding of nonverbal communication as pointing or gaze direction. After this, the child can treat social signals as deliberately communicative. A new step in this development is taken by the age of 5 when the child also will be able to predict and explain other peoples behaviours in terms of mental states (Frith \& Frith, 2007).

Another kind of development from ego-centric to non-egocentric functioning is seen in the development of spatial cognition. In the normal development of orienting strategies, the egocentrically orientation of infants is followed by strategies based on a limit of landmark cues which are dominant until the age of 5. The child knows for example that the door is in front of the later appearing picture on the wall. Later, by the age of 7 , relational strategies begin to develop, enabling children to adopt an observerindependent frame of reference, implying the use of survey. The child can with this strategy understand that since she is looking for the door from an- 
other direction, it is behind the picture on the wall. This strategy is fully developed at about 10 years of age (Lehnung et al., 2001).

Increased efficiency of information processing. Also in this theme, the developing executive functions are of outmost importance. Processing speed is a lower order skill on which efficient information processing rests. There is a gradual increase of attentional capacity and processing speed up through adolescence, possibly with a developmental spurt around 15 years (Anderson et al 2001a). Following the rapid development in early and mid-childhood, the maturation of executive functions continues during late childhood and adolescence, although at a slower rate. Skills of cognitive flexibility and monitoring appear to be stable between 11 and 15, thus maturing prior to adolescence (Anderson et al., 2001a). Since strategic cognitive performance is dependent on executive function, the impact of executive development is revealed through the increasing efficacy in other cognitive areas. Memory capacity, for example, normally increases because of the smoother execution of cognitive operations (Schneider \& Pressley, 1997) and an important increase in the use of elaborative memory strategies that takes place from late childhood to late adolescence (Yeates \& Enrile, 2005).

Growth in knowledge-base. Possession of a deep and well-organized knowledge-base of people, objects, events and ideas enables the child to assimilate new information in a more efficient way. Within an individual, the quality of the knowledge-base inevitably differs from one domain to another, reflecting their personal skills and interests (Ylvisaker 1998).

Ylvisaker's account of normal cognitive development also serves as a summary of functions that are vulnerable in a CTBI. He concludes that many of these areas of development, particularly strategic thinking, non-egocentric thinking, abstract thinking, decentration and efficient use of organizing schemes are associated with the slow and protracted neuroanatomic and neurophysiologic development of the prefrontal areas of the brain. Since frontal lobe function is most often disturbed after CTBI due to diffuse injuries (Vik et al., 2006) or focal injuries (Mendelsohn et al., 1992) the cognitive profile after CTBI often resembles a cognitive profile of a younger child (Ylvisaker 1998).

\section{Brain reserve capacity}

Variations in genetics, previous insults or exposure to neurotoxic agents at some time prior to receiving a brain injury give individuals a different brain reserve capacity (Dennis et al., 2007). The point at which the pathologyburden of a brain insult is such that the brain substance is reduced below a critical level will, therefore, differ from one individual to another. The brain reserve capacity, henceforth referred to as the BRC, refers to the passive capacity of the brain at the time when the person is involved in the traumatic 
accident. In the event of an accident or illness affecting their brain, individuals with higher levels of BRC will be deficit free for longer than individuals with lower levels (Dennis et al., 2007). In persons with more reserve, synapse loss must be more severe before clinical symptoms appear (Stern, 2002). An indirect measure of the brain reserve capacity can be obtained by using diffusion tensor imaging to examine the density in the cortical and subcortical white matter (Dennis et al., 2007), a higher density being indicative of more developed myelinisation. BRC can also be measured in terms of the brain integrity prior to an insult. The integrity of the brain is affected in a negative way under various conditions: repeated brain insults in an animal study resulted in a type of cell damage that was not evident after a single mild injury, and cells in the hippocampus proved to be susceptible to cumulative damage arising from repeated mild traumatic insults (Slemmer, Matser, De Zeeuw \& Weber, 2002). Persons with pre-injury repetitive concussion were more common among adults with severe TBI than among those with mild and moderate TBI (Saunders et al., 2009). Seizures are commonly seen after repetitive concussions (Saunders et al., 2009). Neurotoxic agents can result in significant cognitive impairment, for example from cancer treatment to the central nervous system (Smedler \& Winiarski, 2008; Butler $\&$ Copeland, 2002) and from chronic exposure to alcohol during gestation (Green et al., 2009). Further factors that have been highlighted as having an impact on brain integrity are pre-term birth. A birth-weight under $1500 \mathrm{~g}$ is associated with a risk of having a smaller grey and white matter cortical volume (Nagy et al., 2009), and may result in a neurobehavioural organization different from that of children born at term (Böhm, Lundeqvist \& Smedler, 2010). In addition, genetic defects in children are associated with deficits in the brain regions that are reliant on the neurotransmitters controlled by the deficient genes, thereby reducing brain substrate (Dennis et al., 2007). Neononatal infections in the central nervous system can result in neuropsychological deficits as shown in the study by Englund et al. (2008), where a neonatal herpes virus infection had a negative impact on children's cognitive functions at the long-term follow-up. These are all examples of factors by which the BRC may be taxed.

\section{Cognitive reserve capacity}

In addition to the BRC, people also have a cognitive reserve. Like the BRC, the extent of the cognitive reserve capacity (CRC) differs between individuals and the level of this reserve also influences the outcome of a CTBI (Dennis et al., 2007). Cognitive reserve is according to Stern (2002) the ability to optimise performance through a more efficient use of brain networks or through the ability to recruit alternate brain networks when a more standard approach is no longer operational. While the BRC concerns the passive ca- 
pacity within the available brain networks, the CRC concerns the way in which these networks are used (Stern, 2002). An example of differences in cognitive efficiency when using the same brain structure is presented in an article by Casey, Giedd \& Thomas (2000) who compared the brain activation of children and adults on tasks requiring attention and inhibitory control. The results suggested a similar activation of the prefrontal areas, but with an activation level that was two or three times higher for the children than for the adults; the children still encountered more difficulty in conducting the task. This result exemplifies a higher CRC in the adult group.

In children, the CRC is measured by proxies, some intrinsic to the child, others reflecting relevant environmental influences (Dennis et al., 2007). Fay et al. (2010) reported that children who prior to the injury had a lower cognitive ability had an increased number of symptoms reported after a mild TBI. Pre-injury behavioural functioning has been reported to be a predictor of post-injury behavioural functioning (Taylor et al., 2002; Catroppa \& Anderson, 2008; Fay et al., 2009). The influence of the environment on children's CRC is seen in a study by Yeates et al., (2004), where pre-injury familyrelated environmental factors characterised by a lower socioeconomic status, fewer family resources and poorer family-functioning moderated the longterm social outcome of a CTBI in a negative manner. Dennis et al. (2007) commented on the result of Yeates and his coworkers', by expressing the opinion on that the result can be seen both as a description of the environmental capacity to provide support after the injury, and as support for the hypothesis that privileged environments yield a greater CRC.

\section{Vulnerability versus plasticity}

Recovery of the brain is understood within the concept of plasticity, a prominent feature of the central nervous system that denotes several capacities such as the ability to adapt to changes in the environment and to assist learning (Johnstone, 2009). Plasticity works through modulation of the neurogenesis, through changes in the strength of synapses and through reorganisation of neural circuits (Johnstone, 2009).

Developmental factors play a central role in the outcome after an early brain injury, but there is a debate about whether the immature brain has a greater capacity for plasticity and therefore has better options for recovery than the adult one, or the opposite, whether the immature brain is more vulnerable to insult compared to insults inflicted when older (Anderson et al., 2009c; Taylor and Alden, 1997). Anderson et al. (2009c) summarise the origins of the debate; the plasticity perspective comes from the notion that plasticity is maximal within the central nervous system in early development and argues that the young brain is, therefore, less susceptible to early brain insult (Kennard, 1936). The vulnerability perspective (Hebb, 1947) argues that brain 
insult will affect development differently depending on the age at injury since the cognitive development of a person is dependent on the integrity of particular cerebral structures at certain periods during development.

According to Taylor \& Alden (1997), the literature has provided more support for the vulnerability perspective (see the next section, Age at injury). Conversely, some results considering the outcome of focal injuries lend support to the plasticity perspective. In a review of neuroplasticity following non-penetrating TBI, Levin (2003) reports from longitudinal studies of children with congenital focal brain injuries that an initial lag in development tends to be followed by more typical development. When language functions were impaired, recruitment of homotopic areas in the right hemisphere was associated with recovery. Carlsson (1994) gives another example of the plasticity of focal lesions when showing that early unilateral insult in the left hemisphere supporting verbal functions was followed by a re-lateralization of verbal abilities, thereby preserving verbal abilities and indicating early high plasticity of verbal functions. However, the re-lateralization of verbal abilities was followed by deficits in non-verbal abilities. Lesions in the right hemisphere resulted in impaired non-verbal functions and verbal abilities at a normal level, implying that there are different levels of plasticity available for different cognitive functions (Carlsson, 1994). The initial delay described above by Levin (2003) could be a possible manifestation of the relateralization described by Carlsson (1994).

According to Levin (2003), the literature is more vague concerning neuroplasticity of diffuse axonal injuries. Injury at the time of myelination could disrupt the development of connectivity and therefore diminish the eventual organization of cognitive skills and executive function. Furthering this, Levin (2003) argues that the view of enhanced plasticity might apply to early focal lesions, but has not been supported by studies of early severe and diffuse injury. Anderson et al. (2009c) revealed results that supported the vulnerability perspective for focal injuries as well, since injury before 2 years of age was linked to global and often significant cognitive deficits, while children injured when older performed more closely to normal expectations. Kochanek (2006) argues, without specifying the type of recovery, that the plasticity concept of Kennard might be too broad for CTBI and that an optimal age-window might exist during in which neural plasticity and the associated recovery is most pronounced.

The recent review by Johnston (2009) describes how the mechanisms associated with enhanced plasticity in the developing brain can both contribute to a stronger capacity for learning and result in an increased vulnerability. The capacity to be influenced by the environment is stronger in children than in adults, resulting, for example, in quicker learning of languages. The increased vulnerability, on the other hand, rests on an enhanced excitability across synapses, which increases the plasticity of the developing brain, but it also makes it more vulnerable to damage from drugs, seizures, sensory dep- 
rivation and abuse. Taken together, the greater plasticity of the developing brain does not translate into greater recovery from injuries than the recovery observed when brains are subject to damage at a different part of the life cycle (Johnstone 2009).

\section{Age at injury}

As described by Emanuelson (1999), several physical characteristics increase the vulnerability of a child to traumatic brain injuries. A more vulnerable skeleton and a skull that is relatively large when compared with the size of the body and containing a larger than normal proportion of water in the brain makes the child more sensitive to acceleration, deceleration and violence (Emanuelson, 1999). The cognitive outcome of TBI is also related to age at the time of injury (Koskiniemi, Kykkä, Nybo \& Jarho, 1995; Taylor \& Alden, 1997; Anderson, Catroppa, Morse, Haritou \& Rosenfeld, 2000, 2005; Anderson, Morse, Catroppa, Haritou \& Rosenfeld, 2004; Lehnung et al., 2001; Dennis, Guger, Roncadin, Barnes \& Schachar, 2001; Slomine et al., 2002; Levin, 2003; Donders \& Warschausky., 2007).

Age-related effects have been most evident in comparisons of children younger than the age of 7 with older children and adolescents, and of infants and younger pre-schoolers in a comparison with somewhat older children (Taylor \& Alden, 1997). For mild CTBI, age appears to be unrelated to recovery, while severe injuries received at a younger age are associated with a poorer outcome (Anderson et al 2000, 2005). For example, a severe TBI sustained at a younger age affected the ability to attain word fluency more than a comparable injury in older children, and, furthermore, the long-term recovery of language-abilities took place at a slower rate after severe CTBI at a young age than when they were older (Levin et al., 2001). The recovery of older children from severe TBI is better than that of younger ones, and is more closely aligned to the recovery seen in adults (Anderson et al., 2000).

Skills that mature earlier in childhood may be less impaired of a CTBI than those that develop into adolescence, a difference that reflects the increased vulnerability of emerging versus established abilities (Anderson, Catroppa, Morse, Haritou \& Rosenfeld, 2000). In a study of Anderson and collaborators the vulnerability of emerging abilities was supposedly seen when sustained attention, divided attention and response inhibition, all of them maturing later in development, were found to be more vulnerable than focused attention, reaching adult levels during mid-childhood (Anderson, Fenwick, Manly \& Robertson 1998). Further, in a study comparing mild, moderate and severe CTBI (Muscara, Catroppa \& Anderson, 2008), no differences between groups regarding attention control, a function maturing early in childhood. Conversely, cognitive flexibility, abstract reasoning and goal-setting, all being later maturing executive functions were areas where 
performances differed between the groups. Lehnung et al. (2001) reported that earlier developed spatial orienting strategies were less affected found by a CTBI than later established ones. At a follow-up conducted 4 years after receiving a severe CTBI, (Lehnung et al., 2003) previously established spatial learning skills had been restituted, while more advanced strategies, in the normal development fully functioning by the age of 10 years, were still impaired. A possible explanation of the vulnerability of emerging skills is that injury at the time of rapid myelination can disrupt the development of connectivity and constrain the organization of networks mediating cognitive skills (Levin, 2003).

In a study of children injured between the ages of 3 and 6, no age-related effects were found at an assessment 1,5 month after the injury (Taylor et al., 2008). The result differed from earlier studies that have examined CTBI across wider age-spans. The authors pointed out two possible reasons for their results: the age at injury may be less strongly related to outcome during early childhood. Alternatively, the impact of a younger age at the time of injury may actually become more pronounced with an increasing period of time since the injury (Taylor et al., 2008).

\section{Linear or stepwise recovery?}

The question of whether the relationship between the age at injury and outcome is linear or step-wise is a recent and interesting topic in the CTBI literature (Jacobs, Harvey \& Anderson, 2007; Anderson et al., 2009; Kochaneh, 2006) and is an issue that is also being studied in animals (Kolb, 2004). Brain maturation is stepwise with different periods of alterations of grey matter density, possibly reflecting increased myelination and synaptic and dendritic growth (Gogtay et al., 2004; Lebel, Walker, Leemans, Phillips $\&$ Beaulieu, 2008). Cognitive development is also traditionally described as step-wise (Piaget, 1982), and may be reflecting developmental spurts in the brain. Jacobs et al. (2007) provided support for the existence of a more favourable age for recovery of executive outcome after focal frontal injury: Persons injured between 7 and 9 years of age had better outcomes than the groups comprised of those with a younger and older age at the time of injury and the authors argued that this could be due to the rapid development of executive skills during those ages.

A study by Anderson et al (2009c) evaluated the outcome of 164 children with focal injuries, grouped according to their age at injury into six different developmental periods, progressing from congenital to late childhood. The results supported a linear association between the age at insult and the cognitive outcome. Behavioural outcome, on the other hand, pointed to a different pattern of vulnerability since children injured between ages 7 and 9 performed worse than the group injured between 3 and 6 years old. A study of 
rats (Kolb, 2004) showed support for an age-related step-wise function of the vulnerability of cognitive functions. The results obtained by Anderson et al (2009c) revealing a linear recovery of cognitive functions diverge from other studies that have demonstrated a step-wise recovery of executive and behavioural functions (Jacobs et al., 2007; Anderson et al., 2009c) and from results from animal studies Kolb, 2004). So far, the results on the topic of linear or stepwise recovery seem contradictory.

\section{Gender}

Gender seems to influence outcome after TBI although very few results of the influence of gender have been reported from the children's population. Girls were found to have showed stronger memory function than boys (Donders \& Hoffman, 2002; Donders \& Woodward, 2003), a difference that was not present in a non-injured control-group (Donders \& Woodward, 2003). Concerning cognitive outcome, adult females have been reported to have stronger executive performances than males during acute rehabilitation after moderate to severe TBI (Niemeier et al., 2007). Adult females also had a better outcome than males at their discharge from rehabilitation (Groswasser, Cohen \& Keren, 1998). Another study of adults showed that, while males had better results for visual analytical skills, the overall results indicated a better cognitive recovery for females (Ratcliff et al., 2007). One explanation of the moderating effect of gender could be the impact on secondary injuries, for example, Rogers \& Wagner (2006) reported that adult females, implying females that have reached puberty, might be favoured by the neuroprotective aspects of progesterone, which appears to have the effect of reducing oedema in the damaged brain.

\section{Development after CTBI}

The child with a central nervous system insult faces the normal task of cognitive development and at the same time the abnormal task of formulating an adaptive response to the insult in order to recover functions existing at the time of injury (Dennis et al., 2007). Initial impairments followed by catch-up are a reflection of reorganization, environmental accommodation or development of compensatory strategies by the child. A worsening of impairments with age suggests latent injury effects, arrest of development in the deficit area or reduced capacity of the environment (Taylor, 2004). In this section of development after CTBI, we will start with the recovery of functions and continue with the development of new functions. 


\section{Recovery of functions}

The recovery of cognitive functions after a CTBI was found to occur primarily during the first year after the injury, with little or no recovery of functions being observed in the following 2 to 4 years (Chadwick, Rutter, Brown, Shaffer \& Traub, 1981; Ewing-Cobbs et al., 1997; Yeates et al., 2002). In a recent meta-analytic review, a substantial recovery in intellectual functioning was found in the moderately injured groups at 24 months or longer after CTBI. The recovery was strong especially in the PIQ and processing speed, while the VIQ, attention, working memory, problem-solving and visual perceptual functioning showed no change from earlier impairments (Babikian \& Asnarow, 2009). For the severely injured group, the results indicated a substantial recovery in intellectual functioning, again, stronger in terms of the PIQ than the VIQ. Further, small to moderate recovery was observed in several aspects of executive functioning, processing speed and working memory (Babikian \& Asnarow, 2009).

Reports of the recovery of different cognitive functions are often contradictory, and Babikian \& Asnarow (2009) summarized the possible causes as follows: the characteristics of the TBI itself are intrinsic, such as the heterogeneity of injuries, the influence of social and developmental processes and the capacity of the brain to recover neurocognitive functioning. Different methodological designs also influence the outcome, for example, when patients are categorised according to their age at the time of injury or the period of time which have elapsed since the injury. Finally, different measures of cognitive functions make it difficult to get an overall picture of cognitive outcome and to make valid comparisons between studies involving different groups of patients.

Instead of the almost exclusively used variable-oriented approach of studying the differences and relations on a group-level, Fay et al (2009) used a person-oriented approach to study individual patterns of functional deficits across time. The individual patterns were then related to injury severity. The researchers reported, that the existence of severe CTBI predicted longitudinal patterns of persistent deficits in domains of neuropsychological, adaptive and academic functioning, while a deterioration of function was seen in the behavioural domain. The results also showed that many children with severe TBI did not exhibit deficits in more than one of the domains used to measure outcome from 6 or 12 months post-injury to 4 years post-injury, revealing that variability within the severe group could be missed if results are only studied on the group-level.

Hawley (2003) interviewed the families of 97 children with mild, moderate and severe TBI, admitted to hospital for 24 hours or more. The interviews took place 2 years after injury with a follow up 12 months later. At follow-up, $24 \%$ of the problems were reported to have completely, or almost completely recovered, $14 \%$ had improved, but were still significant prob- 
lems. $54 \%$ of the problems originally reported had stayed the same and $8 \%$ had got worse. Problems most likely to disappear in the moderate/severe group were concerning sleep and epilepsy. Prigatano \& Grey (2008) evaluated the validity of parents' ratings of their children's overall recovery and psychosocial function after TBI. The study showed that parental ratings were related to the severity of the injury, and therefore the results supported the validity of the parental perspective.

\section{Development of new functions}

The impact of a TBI on a child's ability to achieve developmental milestones is the critical factor in determining long-term outcome (Beaulieu, 2002). However, the understanding of the ongoing development of the brain and the cognitive functions after a CTBI is limited. Taylor (2004) summarized the state of knowledge as follows:

"The central aim of most previous research has been to determine the nature and correlates of sequelae. Few investigations have been designed to test the theories of brain-behaviour relations, conceptualizations of the origins of post-injury behaviour or learning problems, or processes affecting development after TBI. As a result we are aware of deficits, such as poor school performance and weaknesses in memory and executive function. But we have limited understanding of the neural bases of these impairments, their developmental implications, and how deficits in different domains relate to one another." (p. 202).

\section{The family's coping with persisting deficits}

Especially for children with moderate and sever injuries, deficits will be persistent, influencing the daily life of the families. Three years after a CTBI, families reported that children with moderate to severe injuries had problems, notably in the areas of attitude to their siblings, clumsiness, compensation, concentration, follow-up, hearing, information needs, lost hobbies and activities, mobility, mood fluctuations, physical problems, schoolwork, school behaviour problems, general school problems, unsympathetic schools and temper (Hawley, 2003).

In a study by Stacin, Wade, Walz, Yeates \& Taylor (2008) injury-related stress among parents with children with CTBI was found to be related to the severity of the injury and to the presence of chronic life stressors. The use of denial as a coping strategy was related to an increase in parental burden and distress. Older age of the child at injury was associated with a greater burden 
and more distress among parents, maybe related to higher expectations of school performance on older children (Stacin et al., 2008).

Savage et al. (2006) describe that families are often helped by being given clear and understandable information about the consequences of the injury their child has suffered and by an explanation of how to cope with these in the everyday life. The families are often under ongoing emotional stress and, therefore, the information must be ongoing, updated and should engage the service providers involved in the child's care. Most TBIs are the result of an accident, and the guilt of not being able to protect their child makes guilt a common burden for the parents. The family may need support to cope with this issue. As a result of the complex connection between injury and outcome, the prognostic information tends to be limited. The parents, therefore, have to cope with the uncertainty surrounding their child's cognitive recovery, and as behavioural changes and cognitive needs become more evident at school and at home, the parents are faced with the challenge of identifying if and how these changes might be related to the injury. If there are no service providers informing the school about how the child has been affected by the injury and how the educational situation can be arranged to suit the child at the time of recovery and during the subsequent periods, the parents are left with the task of informing the school about their child's needs. After the return to school, there is an ongoing need for information to be supplied to new teachers as the child with remaining deficits changes classes and schools. Alternatively, in the absence of such communication, no information reaches the school, and the child is supposed to continue as usual. When an adolescent with remaining deficits moves into early adulthood, the parents have to cope with more problems, regarding questions related to independence and community-integration. A loss of friends and social isolation is reported from the group, with the consequence that these youngsters tend to have a larger reliance upon siblings and family members (Savage et al. 2005).

Persisting deficits influences the daily life, and contrary, family factors also influence the outcome of the child after a CTBI. This is described in a 30 months follow up study by Anderson et al (2006) where children with severe TBI were more likely to have lower socioeconomic status than the groups with moderate and severe TBI. The results were discussed in terms of low performance due to severe injuries possibly being exacerbated by environmental factors. Yeates et al (2005) also found that measures of executive functioning after CTBI were related to socioeconomic functions in the families.

Crosson et al. (1989) described three levels of awareness of deficits, corresponding to the level of compensation of remaining deficits that can be used. The first level is the intellectual one, in which a person has an intellectual understanding of the fact that a function has been impaired. The next level is the one of emergent awareness, in which a person has a capacity to 
recognize a deficit when it is influencing a current situation. The highest level of awareness is the anticipatory one. Here, a person can anticipate situations where deficits will cause troubles, therefore being able to avoid the difficulties in advance. An anticipatory awareness of how to avoid troubles might be of particular importance for parents of children with TBI, since those children have often reduced awareness of strengths and weaknesses in their abilities (Beardmore, Tate \& Liddle, 1999). A statement from Ylvisaker et al. (2007) might explain some of the ineffectiveness of an information session: ventral frontal lobe injury, often seen in TBI and associated with disinhibition and weak reinforcement learning, reduces the capacity to learn from the consequences of previous experiences and to inhibit behaviours based on past consequences. The authors argued for proactive prevention of negative behaviour and systematic facilitation of positive behaviour, thereby rendering the negative behaviours irrelevant. The above mentioned level of anticipatory awareness (Crosson et al., 1989) of the parents then becomes necessary to enable them to provide situations and settings to give their child opportunities to be successful. Helping the parents with identifying deficits and how to prevent negative consequences should be a common goal in rehabilitation.

However, help from health care professionals are often missing after CTBI (Hawley 2006, Slomine et al., 2006). 12 months after injury, 31\% of parents of children with CTBI reported unmet or unrecognized health care needs, with the most frequent type of unmet need being for cognitive services. The top three reasons given for needs to remain unmet were: that interventions were not recommended by doctor, not recommended or provided by school, and finally, costing too much (Slomine et al., 2006). The caregivers with psychosocial problems prior to a CTBI were three times more likely to report unmet needs compared to caregivers to children without preexisting psychosocial problems (Slomine et al., 2006).

\section{The child's coping with persisting deficits}

Beardmore and co-workers (1999) described why it can be hard for a child to become aware of the deficits he or she has accumulated subsequent to a CTBI. In general, small children have a limited and concrete approach to illness, relying heavily on external cues, resulting in a simplified perception of the nature of the brain. Children with a TBI often make a good physical recovery and therefore have a normal appearance, which gives no concrete external cues of their deficits either to them, or to people around them. Children who remember their life before their injury probably also continue to regard themselves as normal children for a shorter or longer time. A direct consequence of the brain injury, in the form of impaired meta-cognition (Hanten et al., 2004), may also decrease the capacity of the child afflicted to 
show awareness (Beardmore et al., 1999). However, the study by Hanten et al. (2004) points at circumstances that can be helpful for meta-cognitive abilities for children with TBI. In the study, children were asked to predict their performance on a word list learning task, and to evaluate the result after the learning-trials. Before the trial, children with severe TBI exhibited a deficiency with respect to anticipating their likely scores compared to both the controls and to those with mild TBI; this discrepancy was smaller after the trial, although the accuracy of the children with severe injuries at anticipating their likely performance remained rather low. The results were discussed in terms of difficulties in integrating meta-cognitive self-knowledge with meta-cognitive monitoring abilities. The results were clarified in terms of the children with severe injuries having a memory of the past that was relatively well preserved, thus helping their metacognitive abilities. In comparison, without earlier experiences, metacognitive abilities were hampered (Hanten et al., 2004). This result can be beneficial in everyday life by showing how circumstances can improve meta-cognition after CTBI. It also exemplifies how the cognitive reserve of the child is influenced by the environment: the metacognitive networks can be used when the questions concerns facts that the child has an experience of.

According to Beardmore et al. (1999), children with a TBI most commonly reported physical problems and memory-related difficulties. They reported having fewer problems than their parents reported them to have and the discrepancy was most frequent in the areas of behaviour and concentration. An information session providing the children with facts about their brain injury and its consequences did not improve their knowledge. Furthermore, the children who had the most limited levels of awareness were also reported to have the highest levels of self-esteem. The low level of awareness was not related to a coping-style of denial. From this perspective, children's awareness of their deficits is a complex matter that needs to be handled with care. According to the results of Hanten et al. (2004), an information session should attempt to refer to concrete situations and activities from the child's previous experiences, to make it as easy as possible for her to relate to and remember the information given.

\section{The school's coping with persisting deficits}

School plays a significant role in everyday life for all children, also after a CTBI. The child's normal teachers are extraordinarily important for the everyday provision of support and compensation, both in the period of rapid recovery and in the long-term perspective. For long-term planning to be secured the schools, and in some cases, the social services, have to be informed both of the present deficits of the child concerned and of how the everyday consequences may evolve in the coming years (Savage et al. 2005). 
Ylvisaker et al. (2005) pointed out that studies have shown that the profile of cognitive and behavioural abilities after injury at a young age often worsens over the years after the injury, instead of improving as might be expected by parents and teachers. Ylvisaker et al. (2005) outlined detailed descriptions of research-based instructional strategies associated with common CTBI impairments, such as organizational impairment, inefficient learning, decreased speed of processing and fluctuating attention. However, many children with special educational needs attributable to TBI are not identified and their needs may not be connected to the injury (Ylvisaker et al., 2005). Reasons for the gap in services were presented as insufficient training of teachers and school psychologists of how to recognise and work with children with TBI (Ylvisaker et al., 2005).

\section{Time since injury}

Subsequent to a CTBI, the most pronounced recovery occurs during the first year after the injury (Chadwick, Rutter, Brown, Shaffer \& Traub 1981; Ewing-Cobbs et al., 1997; Yeates et al., 2002). In their meta-analytic review of neurocognitive outcomes and recovery after pediatric TBI, discussed previously, Babikian \& Asnarow (2009) reported that the group with severe TBI not only failed to catch up with its peers, but also appeared to fall further behind over time. The magnitude of the difference between those with TBI and the controls was also found to increase in studies with longer mean postinjury intervals, a result that was further confounded by the age of the child at the time of injury. The authors believed that this reflected significant and persistent neurocognitive impairments in a subset of children with severe TBI (Babikian \& Asnarow, 2009). Such a variation within the severe group is supported by the results of Fay et al (2009) in their person-oriented study of functional deficits over time in individual children. There was a pronounced variation within the group of the severely injured, covering persons with four persistent deficits to persons with few deficits 4 years post-injury.

The influence of time is discussed further in the section entitled Longterm cognitive outcome.

\section{Age at assessment}

The effects of a CTBI may become obvious at different ages. Results from cross-sectional studies, for example, have revealed that cognitive weaknesses among children with early brain insults are more prominent in older children than in younger ones. It was not clear if this suppression of the normal growth rates relative to peers after an early brain injury reflected deterioration in skills, failure of children to develop at age-appropriate rates, 
or age-related differences in task complexity (Taylor and Alden, 1997). Frontal damage acquired early in life may, for example, exhibit its most prominent sequelae in later childhood when the executive and self-regulating processes associated with the frontal lobe are critical to psychological development (Taylor and Alden, 1997). Outcome can also vary depending on the different expectations that the child or adolescent receives from the environment at various ages. The expectations of those in the child's environment might follow the tracks of a child exhibiting normal development, with expectations that the child's capacity to take on responsibility and develop independence will increase, but these are hard to accomplish with the deficits remaining after a CTBI. In the study of parental burden conducted within the first months of their children's TBI, Stacin et al. (2008) found that a higher age at injury was related to higher levels of parental stress, possibly because of additional concerns relating to a return to school and expectations related to academic performance. The parenting of younger children may render fewer concerns about changes in cognitive functions, which are important for school performance. An alternative reason put forward by the authors is that deficits in cognitive neuropsychological functions may be more apparent in older children, thus contributing to the parental burden of the parents of the children in this age group (Stacin et al. 2008). In a longterm study of outcome after CTBI, Koskiniemi et al. (1995) found that, while half of the group managed school with average results, only a quarter were able to work full-time in adulthood. They argued that special problems may be picked up and addressed through the provision of individual assistance more frequently when a child is at school than might be the case later in life.

\section{Rehabilitation}

According to Ylvisaker (1998), rehabilitation is intended to re-enable people to do what they want to do after an injury or subsequent to some other cause of acquired disability. Rehabilitation is crucial after CTBI in order to foster recovery through neural reorganisation and, thereby, to improve long-term outcome (Beaulieu, 2002). The importance of fostering recovery is most crucial during the early stages after CTBI (Tepas et al., 2009; Beaulieu, 2002). In a review of rehabilitation considerations, Catroppa \& Andersons (2009) cited the description of the three phases of rehabilitation set out by Mazaux \& Richter (1988). In the first phase, the focus is to provide sensory stimulation during coma and arousal states. The second phase focuses on facilitating recovery of impairments and learning to compensate for residual difficulties. The main concern of the third phase is to facilitate re-entry into the community. 
In rehabilitation with children, the developmental aspect is prominent since the expressions of the impairments will vary in different developmental stages. According to Beaulieu (2002), CTBI rehabilitation should address the way development has been affected and consider to what extent development can be expected, given the nature and severity of the injury and age of the child concerned at the time the injury occurred.

Catroppa \& Anderson (2009) described different rehabilitation approaches as follows:

Direct approaches, where lost functions are trained and impaired skills are worked on by providing the maximum relevant stimulation to the person concerned, for example, through attention training (Van't Hooft et al., 2005).

Behavioural interventions, another kind of direct approach, aimed at overcoming cognitive deficits through means such as self-instructional training and the use of a token economy. Emanuelson, von Wendt, Lundälv \& Larsson (1996) recommended behavioural and social training since behavioural problems hampered the readjustment in society at 2-6 years after discharge from early rehabilitation after a severe CTBI.

Environmental modifications and supports, where education of the family, school and community aims at a better understanding of the child's altered abilities, and aims to determine how the child's functioning can be facilitated through changes in the everyday environment.

Psycho-educational treatments directed at the parents have been found to be beneficial. For example, Ponsford et al. (2001) showed that early information provided to children and parents after mild CTBI reduced stress, optimised early management and reduced the frequency of misattributing problems of mild TBI to pre-existing causes. Long-term family function and child outcome are reciprocally interrelated (Ylvisaker et al., 2005).

Family-based interventions. An example of these was the indirect familysupported rehabilitation evaluated in the study of Braga, Da Paz Junior \& Ylvisaker (2005). The program consisted of a two-week long assessment period, with the goal being to work out an individualised manual of interventions, based on simple activities that could be performed back home by the parents. The parents were also involved in coping-groups. After this period, the families continued to implement the programme at home. For 12 months, each family was supported by two case managers who made home visits and if necessary, mobilised other professionals. The evaluation, based on a randomised control design, showed that the children in the indirect familysupported rehabilitation program exhibited significant improvements in cognitive and physical outcome measures, unlike the group with cliniciandelivered hospital based rehabilitation (Braga et al, 2005).

However, there is otherwise a lack of research having been conducted on integrated interdisciplinary models, and with much of the existing research being limited to discipline-specific approaches (Catroppa \& Anderson, 2009). Another exception is the evaluation of a coordinated family and 
community outreach program for children with mild, moderate and severe acquired brain injury and their parents. When compared to a group having only two medical follow-ups, the multi-disciplinary outreach team providing ongoing services was related to significantly higher levels of knowledge about TBI among parents and greater improvements in the school performance of the children (McDougall et al., 2006).

In a review of cognitive rehabilitation for children with acquired brain injury Limond \& Leeke (2005) found the publication rate was lower than one publication per year, resulting in a very limited evidence base. Only one study had sampled more than 10 participants and therefore few generalisations could be made. Comparisons between studies were impossible by heterogeneity in participant variables, treatment variables and assessment/outcome variables (Limons \& Leeke, 2005). Two years later, a review of cognitive and rehabilitation treatment studies by Laatsch et al. (2007) resulted in evidence-based recommendations for the treatment of children and adolescents with acquired brain injury. The authors stated that serviceproviders should consider attention remediation to assist recovery (Van't Hooft et al., 2005, Butler \& Copeland, 2002) and that service-providers should consider involving family-members as active treatment providers in the rehabilitation treatment plan (Braga et al., 2005). As far as the use of information-providing materials was concerned, the recommendation of Laatsch et al. (2007) was that parents or guardians seen in an emergency department would most likely benefit from the provision of an information booklet (Ponsford et al., 2001) clarifying the symptoms of TBI and discussing how the symptoms should be handled. Laatsch et al. (2007) expressed a need for experimentally derived prospective studies with randomly assigned experimental-control groups and masked outcome measures.

\section{Longterm cognitive outcome}

The section of Early cognitive outcome described outcome when most children are back in everyday life, at home and at school, still being in the phase of recovery. In this thesis, the expression long-term outcome implies that most of the recovery has already taken place, but somehow the outcome still changes, moderated by subsequent physical, psychological and social developmental phases. However, there are comparatively few studies that have adopted such a long-term perspective, so little is known about progress made after the first 5 years. The few studies that do exist will be reviewed briefly below.

According to a study by Yeates et al. (2002), children with severe TBI who displayed deficits in neuropsychological functions maintained the deficits in a variety of domains 4 years post-injury. 10 years after moderate and severe CTBI, the intellectual functions of the children afflicted were signifi- 
cantly lower than for controls, especially in tests of verbal IQ, verbal learning and memory, visuo-constructive ability and executive functions (Horneman \& Emanuelson, 2009). Muscara, Catroppa et Anderson (2008) evaluated executive functions 10 years after mild and moderately severe CTBI and reported that the combined moderate and severe group had weaker results than the mild group on several measures of executive functions. An exception was for attention control, which matures earlier in childhood. Long-term studies of the outcome after severe TBI in childhood and adolescence have shown that former CTBI patients display a decreased ability to lead an independent life and poor assimilation in society, despite basic intellectual function having been preserved and regardless of only moderate to mild motor disability (Cattelani, Lombardi, Brianti \& Mazzucchi, 1998; Emanuelson et al, 1998). In a late outcome investigation conducted 23 years after mild to severe brain injury in childhood, intellectual and emotional problems were the most frequent sequelae identified by self-reports. The intellectual complaints included difficulties with learning, memory, intellectual functioning and slowness of thinking (Klonoff, Clarc \& Klonoff, 1993). Quality of life has been found to be negatively affected 10 years or longer after injury (Horneman et al., 2005; Cattelani et al., 1998). For a group of 124 persons, 14 years after CTBI, difficulties were reported to have persisted into adulthood. All groups, mild, moderate and severely injured, exhibited poorer school performance and had employment difficulties. Those in the moderate and severe groups also had an increased risk of having mental health problems, and the severe group reported a poor quality of life (Anderson, Brown, Newitt \& Hoile., 2009). A study of persons who had been severely brain injured at a preschool age (Koskiniemi et al., 1995) found that the outcome in adulthood, defined as the capacity to care for oneself, was poor. Outcome was worse among those who were the youngest at the time of the injury, even if the period of unconsciousness was only of short duration. In a 30-year follow-up study of a group of 27 CTBI patients (Nybo, Sainio \& Muller, 2004), only nine persons worked fulltime, a further two had subsidised jobs and 16 were not working. Neuropsychological functions were evaluated, and preserved cognitive flexibility revealed to be associated with full-time employment. A methodological comment on long-term outcome measures was given by Donders \& Warschausky (2007), saying that longterm follow-up should focus on sensitive neuropsychological tests or standardised ratings of real-life specific skills, such as obtaining of a driver's license, rather than measures of global ability levels.

Even though a number of studies are presented here, the cognitive longterm consequences of CTBI are poorly understood. In comparison to the relatively extensive literature on adult TBI, where significant problems have been described from follow-ups over several decades after the injury, there are only a handful of studies following a group with CTBI up to early adulthood (Anderson, Brown, Newitt \& Hoile, 2009). Taylor (2004) summarised 
the need for long-term studies to be conducted in the future: we have much to learn about patterns of change after CTBI and the processes that underlie those changes. There is a need to know more about how sequelae evolve with age. Furthermore, the patterns of recovery, from the initial manifestations of sequelae to their residual forms or resolution should be studied. Children should be followed for more than 5 years. There is a need for more detailed assessments of components exhibiting change, including: the degree of initial deficit, the rate of catch-up to normative expectations over the first few years after a CTBI, the type of residual deficit and, finally, the long-term changes in residual deficits (Taylor, 2004). One more aim can be added; we need to know if the sequelae change over time, following advancements in neurosurgical CTBI treatment.

There is a lack of studies examining long-term cognitive outcome, a lack of knowledge about how sequelae evolve over time and an absence of or deficiency in the quality of the evaluations of the outcome of children after the international advancements in the neurosurgical care, including those made in Sweden. This was the framework for the choice to make long-term cognitive outcome after a childhood traumatic brain injury the focus of the thesis. 


\section{Present investigations}

\section{Aims}

The main objective of this thesis is to provide new knowledge about the long-term cognitive outcome for more than 5 years after a CTBI. Furthermore, to evaluate if the long-term cognitive outcome of CTBI had been influenced by advancements in neurosurgical care. Additional objectives were to describe Swedish epidemiology and the early process of care, to evaluate if the advancements in neurosurgical treatment had influenced the early outcome and, finally, to study the relationship between early brain injury parameters and early functional outcome.

For study 1, the primary aim was to evaluate how neuropsychological functions progressed over 14 years after a TBI in childhood and adolescence. The secondary objective was to evaluate the psychosocial long-term outcome of those afflicted with a CTBI.

In study 2, the aim was to describe the causes and severity of injury, the process of care from the initial emergency care to rehabilitation, to specify the rate at which rehabilitation and medical follow-up were received in two clinical cohorts, treated in 1987-1981 and 1997-2001, before and after the initiation of the "Lund Protocol". For the group treated in a more structured way after the initiation of the "Lund Protocol", an additional aim was to relate the severity of the brain injury parameters during the acute care stage to the functional outcome at discharge.

In study 3, the aim was is to investigate groups with CTBI who had been treated neurosurgically in 1987-1991 and 1997-2001, respectively, to evaluate if there were long-term cognitive deficits and to see if advancements in neurosurgical care had influenced long-term cognitive outcome. 


\section{Participants in the studies}

The participants in the three studies were drawn from two cohorts of CTBI patients, 10 years apart, as presented in Figure 2.

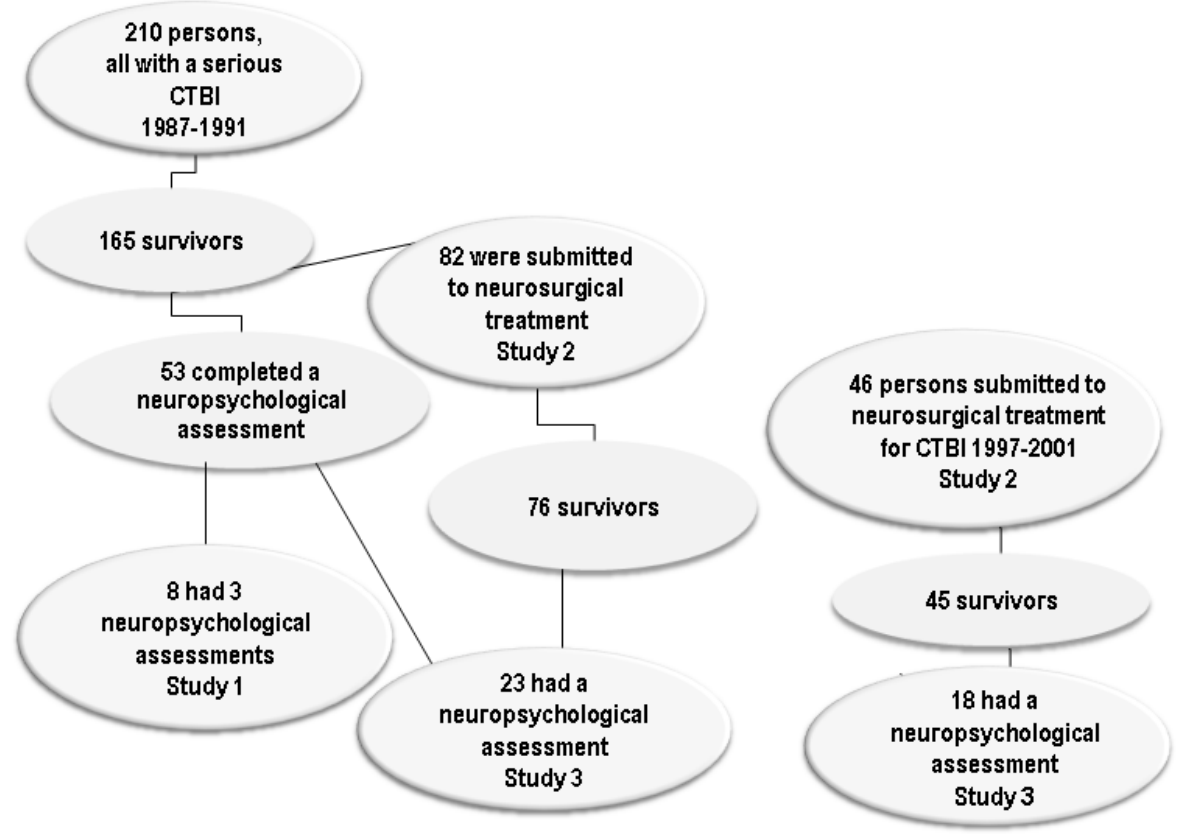

FIGURE 2. Persons with CTBI who were injured between 1987-1991 and between 1997-2001 in the Western Swedish Healthcare Region and their connection with the studies

\section{Participants of Study 1}

The subjects in the first study were part of a patient population of 210 children who suffered serious traumatic brain injury during the period 1987-1991 in the Western Swedish Healthcare Region. Serious injury was defined as one hour or more of unconsciousness and/or neurological or 
neuro-radiological signs of a brain contusion or haemorrhage. The population of the Western Healthcare Region is 1.5 million, with the city of Gothenburg accounting for 500000 inhabitants and the remaining areas being considerably more sparsely populated. In total, 32 of the survivors had received rehabilitation where the first clinical neuropsychological assessment also was conducted. 18 of those patients were included in an outcome-study made 7 years after the CTBI (Emanuelson et al., 1998). Finally, nine of them accepted the invitation to participate in an additional follow-up study (Horneman \& Emanuelson, 2009) conducted 14 years after their TBI. These nine persons had therefore results from three neuropsychological assessments. One patient with a brainstem injury was excluded owing to a restricted ability to participate in neuropsychological testing, and the remaining eight (five females and three males) constituted the study group as indicated in Figure 2. Seven persons were injured at around 15 years of age, and one was 7 years old at the time of the injury. Seven of them had suffered injuries from traffic accidents and one had been injured in a shooting accident. The mean length of coma was 12.5 days (range 2-21) and the median score for the coma depth was 3 on the Glasgow Coma Scale six hours after the injury (range in coma depth: 3-8). They underwent post-injury rehabilitation for a mean time of 244 days (range 30-527). As a group, the patients in Study 1 were considered to be the most severely injured subgroup in this thesis. These eight persons are part of Group 1 in study 2 , since they all had neurosurgical treatment, and Group 1 in study 3 , since they also were part of the follow-up study by Horneman \& Emanuelson (2009).

\section{Participants of Study 2}

Study 2 was based on two groups of CTBI patients admitted 10 years apart. Group 1 originated from the same population as the patients in Study 1, which consisted of 210 children, aged 0-17 years who were injured between 1987-1991 in the Western Swedish Healthcare Region. In this study, we were interested in patients who had received neurosurgical treatment, which meant all patients having been neurosurgically treated on the Sahlgrenska University Hospital. Of the 149 traceable persons, 82 persons had received neurosurgical treatment, defining the first group, Group 1.

In the same healthcare region, between 1997-2001, 46 children, 0-17 years, were admitted for neurosurgical treatment for traumatic brain injury at Sahlgrenska University Hospital. The reason for their referral was unconsciousness, a brain hemorrhage or a contusion. These 46 persons constituted the second group, Group 2. 


\section{Neurosurgical methods used on the two cohorts}

Before 1992, the neurosurgical treatment at Sahlgrenska University Hospital for severe TBI was individualised for each case. Since 1992 and onward, however, neurosurgical care has followed the so-called "Lund protocol" for severely injured patients, with an emphasis on volume-targeted interventions, aimed at controlling intracranial pressure (Grande, 1989, 2006; Asgeirsson et al., 1994). One consequence of this therapy is that the patients with CTBI started to be treated in a more structured manner, taking into consideration the fact that the time taken for brain swelling and blood-brainbarrier damage usually approaches 8-12 days. The change in treatment protocol that was introduced in 1992 gradually changed the grounds for referral to neurosurgical intensive care, with a stronger emphasis on volume-targeted interventions also in patients for whom the full Lund Protocol was not indicated. Therefore, Groups 1 and 2 represent two different cohorts, with different grounds for referral to neurosurgical intensive care.

\section{Participants of Study 3}

Study 3 was based on data from two groups with the joint criterion of being admitted to neurosurgical care for a CTBI, and with a differing criterion being that Group 1 was a population based sample while Group 2 was a hospital based sample. As in Study 2, the two groups with CTBI represent two different cohorts, with somewhat different grounds for referral to neurosurgical intensive care. Previously collected data from a control-group was also used in the study.

Of children who suffered serious TBI between 1987-1991 in the Western Healthcare Region in Sweden, 53 of the surviving patients had agreed to take part in a neuropsychological follow up-study which has been described elsewhere (Horneman \& Emanuelson, 2009). In Study 3, we were interested only in those who had received neurosurgical treatment. Of the 76 CTBI patients who survived the neurosurgical treatment, we had the results of a neuropsychological assessment for 24 persons. One person had too many missing data to be included, so the final group of 23 individuals constituted Group 1. The sub-group that was subject to neuropsychological assessment did not significantly differ from the original group, with respect to gender, $\left(\chi^{2}=0.910, p>0.05\right)$, age at injury $(\mathrm{t}=-.266, \mathrm{df}=97, p>0.05)$, results on the Glasgow Coma Scale (Mann-Whitney U, Z $=-0.452 p>0.05$ ), the number of days of treatment in neurosurgical intensive care $(\mathrm{t}=.092, \mathrm{df}=92$, $p>0.05$ ) or the GOS (Mann-Whitney $\mathrm{U}, \mathrm{Z}=-0.038, p>0.05$ ). Therefore Group 1 is representative of the original group of CTBI patients who between 1987-1991 survived the period of neurosurgical treatment. 
Group 2 originated from the cohort of 46 children and adolescents, aged 0-17 years, who had been admitted to the Sahlgrenska University Hospital for neurosurgical treatment after traumatic brain injury between 1997-2001. The whole group of survivors, 45 in total, were invited to take part in this study and 19 families accepted the invitation. Owing to the severity of the functional deficits, one person could not participate in the neuropsychological testing, and was therefore excluded from the study. The remaining 18 constituted Group 2. Group 2 did not differ from the original hospital-based group of patients, admitted to neurosurgical care, regarding gender $\left(\chi^{2}=0.006, p>0.05\right)$, age at injury $(\mathrm{t}=-.023, \mathrm{df}=62, p>0.05)$, RLS (Mann-Whitney U, Z=-1.121, $p>0.05$ ), GOS (Mann-Whitney U, Z = -0.848, $p>0.05)$ and the number of days of treatment in neurosurgical intensive care $(\mathrm{t}=.068, \mathrm{df}=62, p>0.05)$. We therefore regarded Group 2 as being representative of the original hospital based population of children aged 0-17 who were neurosurgically treated for CTBI in the period 1997-2001. Unfortunately, the comparison with the total group of patients admitted to neurosurgical care was inconsequent since the comparison in Group 1 was based on the original group of patients surviving the period of neurosurgical care. However, only one person died during the period of the neurosurgical treatment, and the inconsequent base for the calculations of the later period may result in minimal bias.

The control-group consisted of 40 healthy individuals originally matched by age and sex with another group with CTBI (Horneman \& Emanuelson, 2009). They were recruited from the national school system and the adult education system and they came from four different areas in Sweden. The inclusion criteria were that they had never visited a hospital for a head injury. The age of Group 2 differed at its assessment from that of Group 1 $(p<0.001)$ and the control-group $(p<0.001)$.

\section{Procedure and statistical methods, Study 1}

Study 1 is a retrospective multiple case study, based on three neuropsychological assessments at three points in time. The assessments took place at a mean time of 1 year post-injury (range 0-3 years), 7 years (range 5-9) and 14 years (range 12-15) post-injury, respectively. Psychosocial data were collected from case-records and through a semi-structured interview at assessment three. The first assessment was the clinical evaluation at the end of an early rehabilitation period. The second and third assessments were conducted within two different follow up studies (Emanuelson et al., 1998, Horneman \& Emanuelson, 2009). Because the setting changed and the time frame was extended, many of the tests differed between the three assessments, with only a limited number of tests providing longitudinal results. 
Six domains of neuropsychological functioning could be compared throughout the three assessments: verbal IQ, verbal fluency, attention and working memory, verbal comprehension, verbal learning and verbal memory. The tests of the domains are presented in Table 1.

TABLE 1. Subtests by cognitive domain in the three assessments of Study 1

\begin{tabular}{ll}
\hline Cognitive domains & Subtests in the three assessments \\
\hline Verbal IQ & I: Vocabulary WISC-III (Wechsler, 1977) \\
& II: SPIQ (Rydberg \& Höhielm, 1974), Peabody \\
& Picture Vocabulary Test (Dunn \& Dunn, 1981) \\
& III: Vocabulary, WAIS-R (Wechsler, 1996) \\
\hline Verbal fluency & I, II, III: FAS, letter S (Tombaugh, Kozak \& Rees, \\
& 1999) \\
\hline Attention and work- & I, II: Digit-span WISC-III (Wechsler, 1977) \\
ing memory & III: Digit span WAIS-R (Wechsler, 1996) \\
\hline Verbal & I, II, III: The Token Test (De Renzi \& Faglioni, \\
comprehension & 1978) \\
\hline Verbal learning & I, II: Verbal learning 10 words, immediate correct \\
& recalls (Christensen, 1984) \\
& III: Rey Auditory Verbal Learning Test; imme- \\
& diate correct recalls (Geffen, Hoar, Hanlon, Clarc \\
& \& Geffen, 1990) \\
\hline Verbal memory & I, II: Verbal learning 10 words, delayed correct \\
& recalls (Christensen, 1984) \\
& III: Rey Auditory Verbal Learning Test; delayed \\
& correct recalls (Geffen, Hoar, Hanlon, Clarc \& \\
Geffen, 1990)
\end{tabular}

\section{Procedure and statistical methods, Study 2}

Demographic data were gathered retrospectively from patient charts. The classification of injuries and diagnoses was performed according to the ICD 9 (800-804, 851-854) during 1987-1991 and the ICD10 system with S06 codes for head injuries during 1997-2001. The results are primarily presented descriptively. Group differences were tested with T-tests for interval scaled data, and with the Mann-Whitney U test for ordinal scaled data, with the level of significance set at 0.05 . In Group 2, individual profiles of the parameters relating to the severity of the brain injury were subject to a hierarchical cluster analysis in SPSS, identifying clusters, or subgroups, of patients characterized by similar profiles. We regarded Group 2 as a popula- 
tion since it covered a 5 year period and included all the $0-17$ years old that were admitted to surgical care for a traumatic brain injury, in the second largest health care region in Sweden. The brain injury parameters were: days of care in the neurosurgical ward, hours of care in a respirator and level of consciousness measured by RLS. We took squared euclidean distances as the measure of the similarity of the profiles and used the Ward method to detect the number of clusters. The determination of the optimal numbers of clusters was primarily based on the theoretical meaningfulness of the solution and a striving to avoid a solution resulting in a sudden drop of the explained variance. The solution was validated by running a complementary cluster analysis, using Complete Linkage as an alternative method of detecting clusters. This is a test of whether the result depends on the method or on the actual data and is therefore, a validation of the analysis.

\section{Procedure and statistical methods, Study 3}

The neuropsychological assessments were made by trained psychologists. The mean time to have elapsed since the injury was 13 years for Group 1, and 6 years for Group 2. 18 subtests were grouped into cognitive domains, presented in Table 2. The mean test-age was 23 years in Group 1, and 17 years in Group 2. In order to compensate for systematic differences in the assessment age between the groups, all results were transformed to T-scores, based on age-adequate norms gathered from the references attached to each test above. Before the analysis, the distributions were examined for assumption violations. Outliers with T-values of less than $10(<-4$ standard deviations, sd) were temporarily withdrawn; mean-values and standard deviations were calculated excluding these cases, and then the outliers were rescored to two standard deviations from the mean and included in further analyses. A multivariate analysis of variance was performed to investigate group differences on cognitive domains and subtests. Preliminary assumption testing was conducted for all subtest-variables. Some violations were noted with regard to normality equality, error variance and equal variance and therefore Pillai's trace was used. In order to reduce the risk of Type 1 errors, the Bonferroni correction of alpha levels was used when comparing group differences for the cognitive domains. For a measure of the strength of the results, partial eta squared, $\eta_{\mathrm{p}}^{2}$, was presented as well. 
TABLE 2. Subtests by cognitive domain in Study 3

\begin{tabular}{ll}
\hline \hline $\begin{array}{l}\text { Functional } \\
\text { domains }\end{array}$ & Subtests \\
\hline $\begin{array}{l}\text { Verbal } \\
\text { Intellectual }\end{array}$ & $\begin{array}{l}\text { Vocabulary WISC-III }{ }^{1} / \text { WAIS-R }{ }^{2} \\
\text { Rey Auditory Verbal Learning Test }\end{array}$ \\
& $\begin{array}{l}\text { Rey Auditory Verbal Learning Test, immediate retention } \\
\text { Rey Auditory Verbal Learning Test, delayed recall }\end{array}$ \\
& Rey Auditory Verbal Learning Test, recognition \\
& Verbal Fluency Test ${ }^{5,6}$ (letters F, A, S)
\end{tabular}

\section{Results of Study 1}

The cognitive results are presented separately for each domain below.

At assessment three, 14 years after the injury, six subjects obtained a verbal IQ below that obtained in their earlier assessments. Compared to norms, four subjects performed between -1 and -2 sd, and 2 subjects performed below -2 sd. 


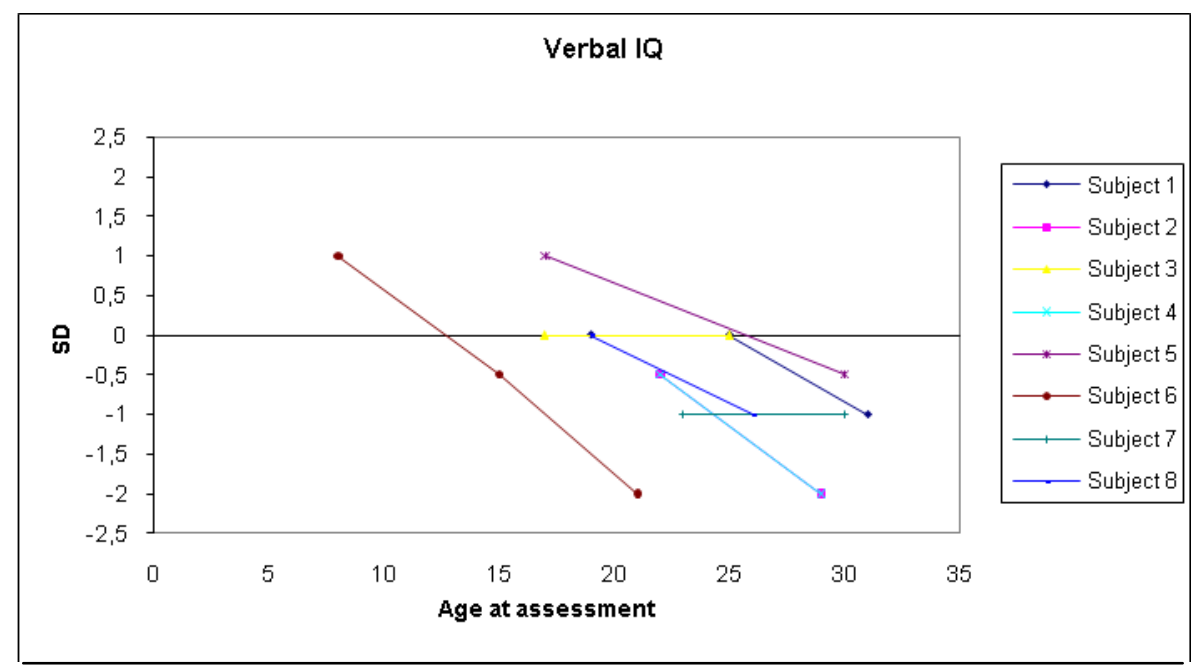

FIGURE 3. Age at the time of the assessments plotted against the verbal IQ performance

The performance in the domain of verbal fluency seemed stable for five subjects and increased after the first assessment for three subjects. At the third assessment, the test did not differentiate within the group.

The result for the attention and working memory domain showed a low performance level for four subjects on assessments two and three, $-1.5 \mathrm{sd}$, or lower for half the group in the third assessment. On the whole, the performance over time seemed to be stable.

In the domain of verbal comprehension, the performances suggested a stable function over time.

Results on Luria's verbal learning test were evaluated against a cut-off score for pathological performance, $-1.9 \mathrm{sd}$, according to which only one patient showed impairment. According to Rey Auditory Verbal Learning Test, at the last assessment, five persons performed below $-1.5 \mathrm{sd}$ and two of them below -3 sd.

In the domain of verbal memory, six persons had results that deteriorated over time, and the other two showed slight or clear improvements.

It was not possible to discern any age at injury effect, primarily due to restriction of range.

A summary of the cognitive results reveals that a weakening of the verbal IQ was seen over the three assessments and verbal learning was the cognitive domain in which the most pronounced impairments were recorded. Further- 
more, the group's performance in the attention and working memory domain at assessment three were low. As for life situation and adaptation, three of the eight subjects had gone straight from a school situation without special education provisions or adjustments to what was effectively an early retirement.

\section{Results of Study 2}

In the epidemiological study, the gender distribution showed that boys were in the majority in both groups, $62 \%$ in Group 1 versus $67 \%$ in Group 2 . The mean age at the time of injury in Group 1 was 9.9 years (range $0.5-17$ years), and it was 11.0 years (range $2-16$ years) in Group 2, a nonsignificant difference. Traffic accidents were the most common cause of injury in both groups, however in Group 1, most of the children had been injured while bicycling, while the traffic victims in Group 2 were mostly pedestrians. The Glasgow Coma Scale indicated a more severe injury level in Group 1 (Mann-Whitney $\mathrm{U}, \mathrm{Z}=-2.964, p=0.003$ ). The mean length of care was 18.7 days for Group 1, and 14.5 days for Group 2. No significant differences were noted regarding the severity parameters, in terms of the number of hours of care in a respirator and days of care at the neurosurgical unit. Regarding neurosurgical procedures, 'no procedures' (38\%) was the most common alternative for Group 1, and intracranial pressure monitoring was the most common alternative for Group 2 (37\%). By comparison, the rate of ICP monitoring was $13 \%$ for Group 1 which was markedly lower than in the later period. In Group 1, four children died during the period of intensive care and two children died during the period of rehabilitation, which gives a mortality of $8 \%$. In Group 2 , mortality was $2 \%$ since one child died during the period of intensive care. In both groups, the measure of functional outcome, GOS, had the median of 5 at discharge, implying resumption of normal life even though there may be minor neurological and psychological deficits that might need compensatory actions. The result on GOS revealed no significant difference between the groups. The rate at which rehabilitation was provided was equally low in both groups, $27 \%$ versus $33 \% .58$ 
children in Group 1 and 30 children in Group 2 did not receive any rehabilitation. Of those, 14 children in Group 1, 18\%, did not receive medical follow-up either, and 15 children, 33\% in Group 2 did not receive either rehabilitation or a medical follow-up of any kind.

In Group 2, treated according to the more structured protocol, we investigated individual profile subtypes of brain injury severity parameters. By application of a cluster analysis, five clusters - that is five profile subgroups came out as a suitable solution explaining 79.6 per cent of the total error sum of squares. The validity check by the cross-method stability turned out in the same five cluster solution, thereby further supporting our solution.

When checking the relation between cluster profiles and the clusters median level of GOS, the result indicates that the length of care and the length of time being cared for in a respirator had a stronger relationship with outcome than did the level of consciousness according to RLS, when the two lengths of care-measures differed from the levels on the RLS. An example of this is shown in Figure 3, 4 and 5 where the RLS differs from the two other brain injury parameters. Despite a deeper unconsciousness at admission, Cluster 1 had a median of 5 on GOS, implying a good recovery; the patients have been able to return to a normal life, even though there may be minor neurological and psychological deficits that might need compensatory action. Cluster 5 had an almost normal level of consciousness, while the length of care and time in respirator were somewhat high and had a GOS of 3.5, corresponding to dependence of daily support by reason of mental or physical disability. A similar situation was seen in Cluster 3, although the level of the mean RLS corresponds to a severe injury. According to the Z-values and the variation within the group, the length of care and time spent in respirator corresponds better to the low outcome score of this cluster, GOS 3.5.

Cluster 1, consisting of eight persons, had a short length of care and time in a respirator but an RLS that indicated deeper unconsciousness. The mean length of care was 6 days, and the mean length of care in respirator care was 19 hours, the mean RLS was 6.1: 'unconscious, stereotype flexion movements on pain stimulation'. Looking at the outcome in Cluster 1, the median of the GOS was 5. Four patients received rehabilitation, and four did not. 


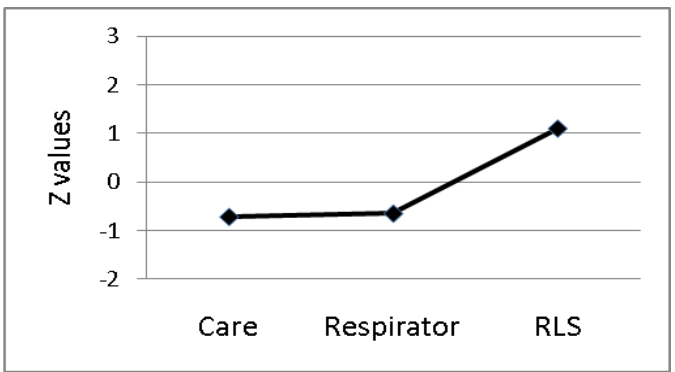

FIGURE 3. Profile of the brain injury parameters for Cluster 1

Cluster 5 was comprised of four patients who received a somewhat high level of length of care and time spent in the respirator and had a low RLS equivalent to an almost normal level. The mean length of care was 22 days, mean care in a respirator was 408 hours and mean RLS was 1.5: almost fully alert. The median GOS was 3.5 and none of the patients had rehabilitation.

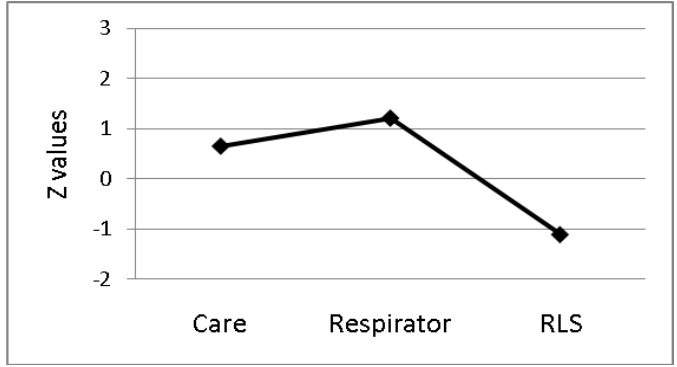

FIGURE 4. Profile of brain injury parameters for Cluster 5

Cluster 3, with four patients, had a long length of care and time in a respirator, and low RLS equivalent to light unconsciousness. The mean length of care was 42 days, mean care in a respirator was 618 hours and mean RLS was 4.3: unconscious, localises but does not ward off pain. The median GOS was 3.5, two patients had rehabilitation and two had no rehabilitation.

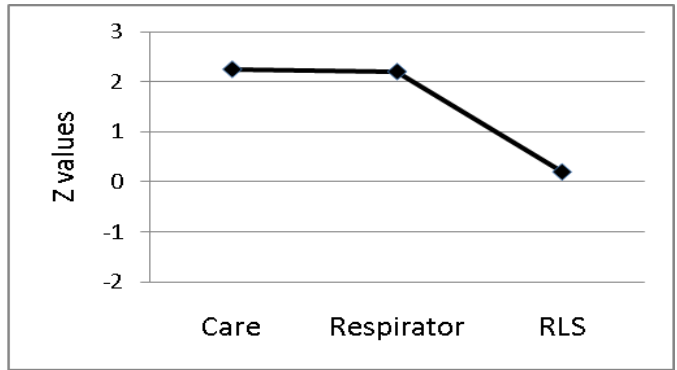

FIGURE 5. Profile of brain injury parameters of cluster 3 
When the Z-values of length of care, time in respirator and level of consciousness were similar, as in Cluster 2 and Cluster 4, seen in Figure 6 and 7, they appeared to correspond to the level of GOS.

Cluster 2, holding 23 patients, had a short length of care and time in a respirator and a low RLS. The mean length of care was eight days, mean care in a respirator was 30 hours and the mean RLS was 2.5: drowsy or confused, responsive to light stimulation. The median GOS for the group was 5. Five patients had rehabilitation, and 18 had no rehabilitation.

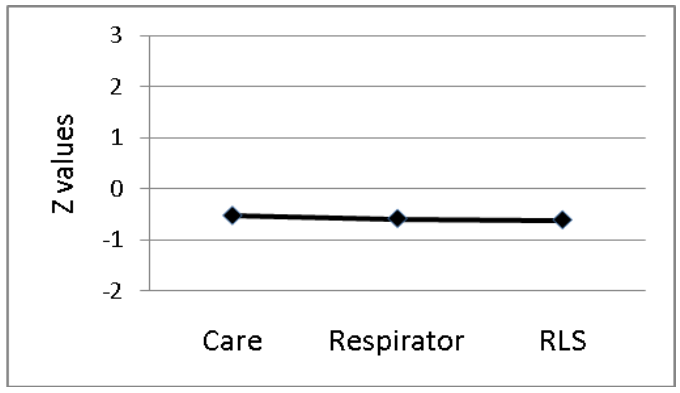

FIGURE 6. Profile of brain injury parameters in cluster 2

Cluster 4, comprising 7 patients, had a fairly high level of length of care and hours in a respirator and an RLS equivalent to deep unconsciousness. The mean length of care was 25 days, mean care in a respirator was 305 hours and mean RLS was 6.6: unconscious, stereotype extension movements on pain stimulation. The median GOS was 4, four patients had rehabilitation, and three had no rehabilitation.

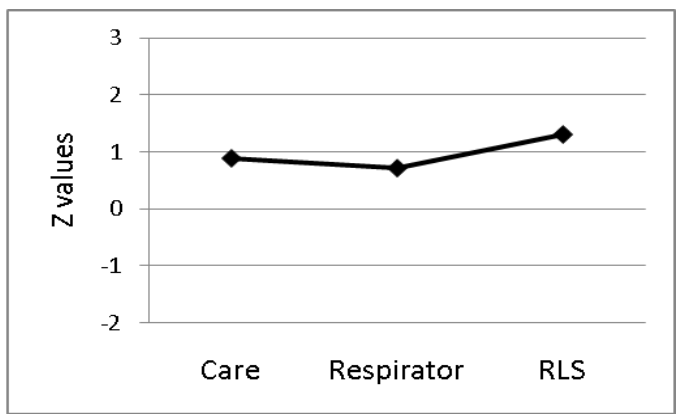

FIGURE 7. Profile of brain injury parameters in cluster 4 


\section{Results of Study 3}

The comparison between the cognitive functions of the groups with CTBI and the controls revealed significant differences, with the controls having significantly stronger results in all four cognitive domains when compared with Group 1, and in three of the domains when compared with Group 2. Long-term cognitive deficits of similar magnitude and character were observed in both groups with CTBI. Compared to the controls, the abilities of the participants were especially low where executive functions, verbal memory and verbal intellectual functions were concerned. The results for the four domains are shown in Figure 8. The two CTBI groups differed significantly only in the executive domain, where Group 2 performed worse than Group 1 $\left(\mathrm{F}=6.713, p=0.001, \eta_{\mathrm{p}}{ }^{2} 0.33\right)$.

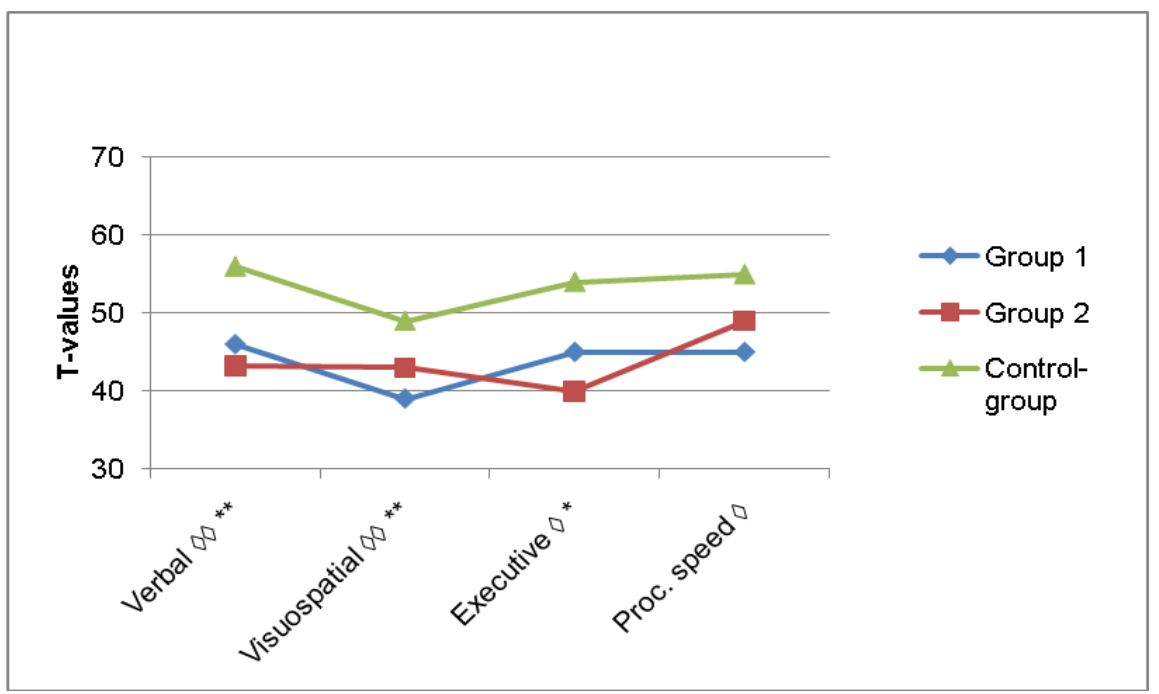

Sign difference group 1 and controls $p<0.008: \diamond \diamond, p<0.017: \diamond$

Sign difference Group 2 and controls $p<0.008$ : **, $p<0.017$ : *

FIGURE 8. Results for the two groups with CTBI and the controls for measures in the cognitive domains of Verbal intellectual functions, Visuospatial intellectual functions, Executive functions and Processing speed

The results for the memory domains indicated larger differences between the control-group and the CTBI groups for the verbal memory functions than for the visuospatial memory functions. This is seen in Figure 9, which exhibits the same information as Table 6 in the article of Study 3. 


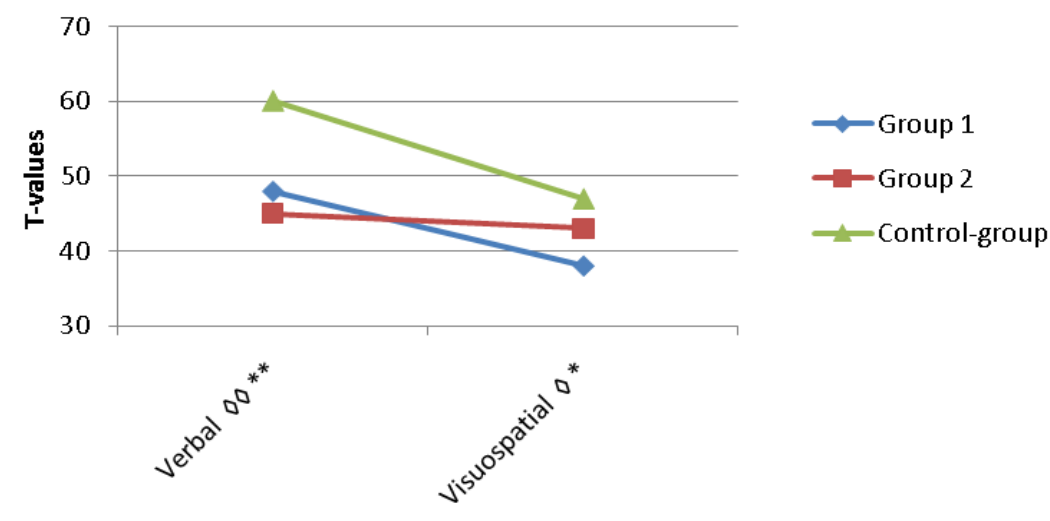

Sign difference group 1 and controls $p<0.012: \diamond \diamond, p<0.017: \diamond$

Sign difference Group 2 and controls $p<0.012: * *, p<0.017$ : *

FIGURE 9. Results for the Verbal memory domain and the Visuospatial memory domain for two groups with CTBI and the controls

When examining individual subtests, there were significant differences between Group 2 and the controls in 14 of the 18 subtests. The control performed significantly better than Group 1 on 17 of the 18 subtests. The subtest results for the two groups with CTBI were also compared, with only one significant difference being found, on TMT B $(\mathrm{F}=12.384$, $\mathrm{df}=1, p=0.001$, $\left.\eta_{\mathrm{p}}^{2}=0.24\right)$. 


\section{General discussion}

The aims of this thesis were:

- to provide new knowledge about the long-term cognitive outcome after CTBI

- to evaluate if the advancements in neurosurgical treatment have influenced the long-term cognitive outcome and the early overall outcome after a CTBI

- to describe Swedish epidemiological features and the early process of care after suffering a CTBI

- to study the relationship between early brain injury parameters and early functional outcome.

Taken together, the results show that former CTBI patients, who received neurosurgical treatment, have significant deficits in most cognitive domains when compared to non-injured persons at long-term follow-up. The longterm cognitive follow-up of the cohort injured between 1997-2001 comprised of patients who received a more structured neurosurgical treatment than the other cohort injured between 1987-1991 treated neurosurgically in an individualised manner. In Group 2, treated in the later period, abilities were particularly low in executive function, verbal memory function and verbal IQ. The cognitive results of the groups suggest a better long-term recovery of visuospatial cognitive abilities and a significant risk of deficits in long-term verbal intellectual abilities and executive functions after serious CTBI. There was no significant difference in the rate of mortality for the two cohorts, and both groups had an early outcome corresponding to GOS 5, also with no significant group difference. The rate at which rehabilitation was undertaken was equally low in both groups, $27 \%$ in Group 1 and $33 \%$ in Group 2. Of those who did not receive rehabilitation, half received no medical follow-up either. The results suggest that current arrangements for referral to rehabilitation and for follow-up of children post-injury have not improved and are still not sufficient. The evaluation of the relationship between early brain injury parameters and early functional outcome indicates, that the length of care in the intensive care unit and length of care in a respirator may have a stronger relationship to outcome than does the single measure of the level of consciousness, RLS, at admission. The latter result is, however, quite explorative in nature, and must be replicated before even tentative conclusions are drawn. 


\section{Verbal and visuospatial intellectual functions}

Early post-injury results obtained using the WISC performance scale are often low owing to diminished functions of motor control and response speed (Slomine et al., 2002). Verbal intellectual functions often appear to be the strongest cognitive abilities in the initial phase after injury, and are sometimes used as a measure of a person's pre-injury intellectual ability (Slomine et al. (2002). This is the base for the interpretation of the results in Study 3 . There it was claimed that recovery most likely had occurred in visuospatial intellectual functions whereas there were obvious long-term deficits in verbal intellectual functions. In Study 3, even though abilities related to verbal memory and verbal IQ were especially low, both of the performances of the visuospatial and the verbal domain are significantly lower compared to performances of the controls. Since early results of visuospatial abilities, measured by the WISC performance scale are often initially low, and verbal abilities, often initially spared, these results indicate a better recovery of visuospatial functions and a long-term vulnerability of verbal intellectual functions at a long-term follow-up. This knowledge has not been reported at this length of follow-up.

Study 3 was based on comparisons between the CTBI groups and the controls. The control group has a somewhat surprising profile of cognitive test results, as illustrated in Figure 8. This may be a reflection of the fact that the various test norms have been collected in different populations, and at different points in time. Therefore, there is no basis for analysing the profiles of the cognitive performance. Since the control group and the groups with CTBI were judged by the same norms, the comparison between the groups is not biased by the norms. The comparison between the groups was the main focus in this study.

Study 3 suggested a better recovery of visuospatial intellectual functions. These findings are both supported by, and differ from, earlier reports. Earlier studies of long-term cognitive outcome have shown deficits on a similar level of visuospatial IQ and verbal IQ (Ewing-Cobbs et al., 1997, Cattelani et al., 1998). Some studies have reported visuospatial intellectual abilities as the ones that were most negatively affected by CTBI. This result was based on follow-ups at 1 year after injury (Catroppa \& Anderson, 2002) and 2 years after injury (Chadwick, Rutter, Shaffer \& Shrout, 1981b). In the latter study, that of Chadwick et al. (1981b), the group of children with head injuries had a verbal IQ score that was almost equivalent to that of the controls at the follow-up that took place 1 year after their injury, and therefore the verbal IQ part was excluded at the next follow-up, 2 years post-injury. Van Heugten et al. (2006) also excluded measures on verbal IQ at a followup made at nearly 7 years after the patients' discharge from rehabilitation; they reported most problems in the domains of attention, memory and executive functioning. Other studies of those who have had a CTBI have reported 
a better recovery for non-verbal skills, like visual perception, visual memory, visuo-motor skills and constructional abilities 1 year after the injury (Anderson et al 2000), 30 months after the injury (Anderson et al 2005) and 5 years after the injury (Anderson \& Catroppa, 2007) and have reported that verbal intellectual abilities may be at risk of long-term persistent deficits (Anderson et al., 2000, Anderson \& Catroppa, 2007). Our results are more consistent with the latter. The discrepancy in the results is a good example of what Babikian \& Asnarow (2009) summarized when they reported on the inconsistencies between studies when it came to the nature of neurocognitive impairment and recovery following CTBI (page 20). The divergent results in the studies presented above may be attributed to variations in the time to have elapsed since the injury and in the outcome measures, making it difficult to obtain an overall picture of cognitive outcome. The studies reporting the greatest negative impact of a severe CTBI on visuospatial functions are based on results from the first year after injury, while results supporting a better recovery of visuospatial skills come from studies where longer time had elapsed since the injury. Our results are further supported by the metaanalytic review of Babikian \& Asnarow (2009), where measures both from the moderate and severe groups show a substantial recovery in intellectual functioning, stronger in PIQ than VIQ, between 0-5 months and 24 months or more post-injury.

In Study 1, the longitudinal verbal IQ, verbal learning and verbal memory showed signs of decline over time. One aspect that might be helpful when evaluating recovery and development in longitudinal studies is presented by Anderson, Catroppa, Morse, Haritou \& Rosenfeldt (2009b), who points out the importance of taking into account the fact that IQ scores reflect developmental changes. Thus, if both normal development and recovery occur, a positive IQ gradient would be present over the time after the injury, however, when only recovery or development takes place there would be a flat trajectory; and in the absence of both development and recovery, a negative gradient would be exhibited. Our results in Study 1 reveal that, as far as verbal learning is concerned, half of the people concerned exhibited a negative gradient, while the majority were found to have a negative gradient for verbal IQ. This indicates that development has at best slowed, at worst been arrested. Negative trajectories could probably even reflect the loss of functions. We interpreted flat trajectories to be representative of a stable situation and assume, in line with this discussion, that development has continued, but on a lower level.

The challenge for neuropsychologists is to identify problems in behaviour, adaptive skills and learning, and to explain how these problems arise and to recommend effective interventions (Taylor, 2004). However, placing the emphasis on the problems could explain why it is so much easier to find results on impairments than on recovery and those functions that have been spared after a CTBI. Knowledge about spared functions is important for 
opening up for initiating effective compensatory interventions, in addition to which, knowledge about recovery can improve our understanding of the process, and thereby provide the people struck by injury with hope.

\section{Vulnerability of verbal memory functions}

In Study 1, verbal learning was the cognitive domain that exhibited the largest impairments, with results below -3 standard deviations. In Study 3, the difference between the groups with a CTBI and the controls was more pronounced for verbal memory than for visuospatial memory. The vulnerability of the long-term verbal memory is supported in the literature (Catroppa \& Anderson, 2007; Anderson et al., 2005; Catroppa, Anderson, Ditchfield \& Coleman, 2008). Further, Donders and Giroux (2005) reported a relation between prolonged coma after a CTBI and a weak result in a verbal learning and memory test similar to the one we utilized, the California verbal learning test. In the discussion in Study 3, it is argued that the diminished function of executive control, which is common after CTBI (Levin \& Hanten, 2005), may negatively affect the learning strategies that people adopt (Yeates \& Enrile, 2005) and, further, that normal brain development, with a late maturation in the frontal and temporal lobe (Gogtay et al., 2004) and ongoing development of memory strategies (Yeates \& Enrile, 2005; Schneider \& Pressley, 1997), will gradually enlarge the gap between the people afflicted and uninjured people. Attention, working memory and verbal learning are dependent on the integrity of the frontal lobes however these are the parts of the brain most frequently involved in residual brain lesions after a childhood TBI (Mendelsohn et al., 1992).

The standardised norms of the subtests in Study 3 support the notion of a late maturation of verbal functions, since there are quite large differences between the ages at which performance reaches the highest levels in the different subtests (Wechsler, 1996; Geffen, Hoar,Hanlon, Clarc \& Geffen, 1990; Meyers \& Meyers, 1995; Spreen \& Strauss, 1998; Yeudall, Fromm, Reddon \& Stefanyk, 1986; Bornstein, 1985; Birkenkamp \& Zillmer, 1998 \& Boll, 1993). The subtests in the verbal intellectual domain are the last to attain a plateau in the development as seen in Figure 10. In the domain of processing speed, the highest levels of functioning are attained in adolescence. As discussed earlier, the norms of these subtests are based on different populations and determined at different points of time, and no meaningful profiles of cognitive performances can be evaluated. Therefore, only the large differences can be informative in this figure, indicative of a prolonged developmental period for verbal intellectual functions. 


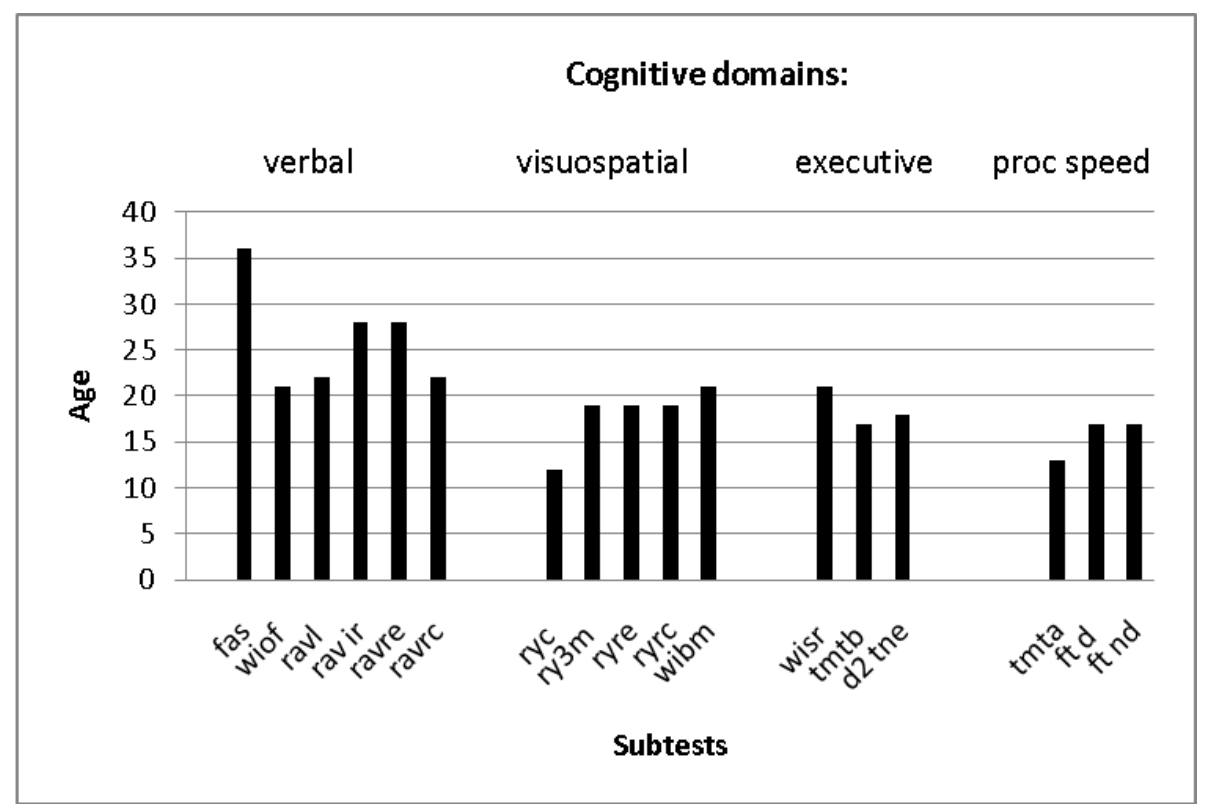

FIGURE 10. Ages when development plateaus according to the published norms of the subtests within the four cognitive domains of Study 3

The results of long-term memory impairments after CTBI are consistent with studies on hippocampal brain function. The hippocampus - a temporal structure important in memory processes - is vulnerable to TBI through both direct and secondary causes (Archinegas et al., 1999). The hippocampus is particularly vulnerable to direct injury owing to its position in the skull, because it can be abraded against lower parts of the scull. Disrupted cholinergic functions arising from direct mechanical forces or diffuse axonal injury can also diminish hippocampal function (Archinegas et al., 1999). The effects of CTBI on brain growth and hippocampal volume might extend into adulthood (Tasker et al., 2005).

\section{The connection between outcome and received rehabilitation}

Evaluations of the impact of rehabilitation have shown significant improvements in cognitive outcome (Braga et al., 2005; van't Hoft et al., 2005), physical outcome (Braga et al., 2005), greater improvements of the children's school performances and higher levels of knowledge about TBI among parents (McDougall et al., 2006). Evidence-based recommendations have been made for the treatment of children and adolescents after an acquired brain injury (Braga et al., 2005; van't Hoft et al., 2005). Service- 
providers should consider attention remediation to assist recovery and claim that service-providers should consider involving family-members as active treatment providers in the rehabilitation treatment plan (Laatsch et al., 2007).

In Study 2, we saw that the amount of rehabilitation received and the follow-up procedure were equally low in both groups, even though important advancements in the neurosurgical care had occurred. In the earlier period, $27 \%$ of the group were admitted to rehabilitation. Of those that did not receive rehabilitation, $24 \%$ were left without medical follow-up. In the later period, 33\% were admitted to rehabilitation and, of the remaining patients, only half of them had a medical follow-up. The results of Study 3 showed that the long-term cognitive function had the same level as the group treated in the later period. The literature in the last few decades has provided an increasing number of reports concerning the cognitive and behavioural deficits after CTBI, and one might therefore expect an increase in the rate of rehabilitation. Obviously, there is a discrepancy between research and clinical practice.

We have received reports of favourable outcome after TBI treatment with intra-cranial pressure targeted therapy (Wahlström et al., 2005), but even in the highest level of the GOS, minor neurological and psychological deficits that might need compensatory action are present. Almost two decades ago, Michaud, Rivara, Grady and Reay (1992) stated that an assessment score of 'good recovery' according to the GOS at the time of a patient's discharge from acute care is likely to be an overestimate, since subtle neuropsychological or neurobehavioural changes are not likely to have been detected before a patient's discharge from hospital.

In the evaluation of the relation between early brain-injury measures and early functional outcome, we found that the level of consciousness at admission as measured by RLS may be a weak predictor of early functional outcome when differing from the measures of length of care and length in respirator. The result in the five-cluster solution was supported by the high amount of variance explained and by the validity check using an alternative method of identifying clusters. However, the result is based on a limited number of persons and needs to be replicated. A search in the literature has revealed that measures incorporating several data points from several different measures (for example repeated measures of consciousness or the taking into account the various measures involved when decisions about the duration of the length of care provided to a patient) might be more reliable than measures based on more limited data, such as one measure on the RLS or the GCS (Massagli, Michaud \& Rivara, 1996; Parslow, Morris, Tasker, Forsyth \& Hawley, 2005). Secondly, predictors of the early outcome might not be the best predictors of the later outcome (Massagli et al., 1996; Niedzwecki et al., 2008). A single measure on the RLS may well serve an important role during acute treatment, but yet not predict long-term outcome. 
According to the extensive evaluation of parental ratings conducted by Hawley (2003) and the evaluation of unmet needs by Slomine et al. (2006), follow-ups should be made available to the entire group of patients with a CTBI, that is mild to moderate, when the families notice TBI-related alterations in everyday functions, or in the event that the health professionals know that soon everyday life, or up-coming developmental paths, might be influenced by the TBI. The final evaluation of outcome after a moderate to severe CTBI should not be conducted before adulthood.

\section{Methodological challenges}

Long-term cognitive outcome in Study 3 was evaluated with the common variable-oriented approach, with the results being examined on a group level. In that study, the mean-values of the groups showed that there are significant cognitive differences between the group of uninjured controls and the groups with a CTBI, and according to the levels of the partial eta squared these differences are often quite strong. However, the standard deviations of the cognitive variables of the groups are large, implying heterogeneous groups. According to Chapman (2001), the extreme heterogeneity in the population of those with a CTBI is one of the most consistent findings in the literature on CTBI and it is important to recall that group results do not predict the recovery of individuals over time. An example of this is seen in the study of Tasker et al. (2005) conducted 5 years after severe CTBI. The majority of a group who had had early monitoring of the intracranial pressure over more than three days had no evidence of cerebral atrophy. A sub-group, with a longer period in intensive care, had a smaller than expected volume of the occipito-frontal head circumference, exhibiting white matter loss and disproportionate hippocampal atrophy. In Figure 11, the results of Study 3 in the domain of verbal memory are shown, revealing that several persons in the group had performances that did not differ from an average to low performance of the individuals in the control group, while others exhibited performances that were quite low, and yet more had results that implied quite substantial deficits. The marked variation within the group is not explained by the study. 


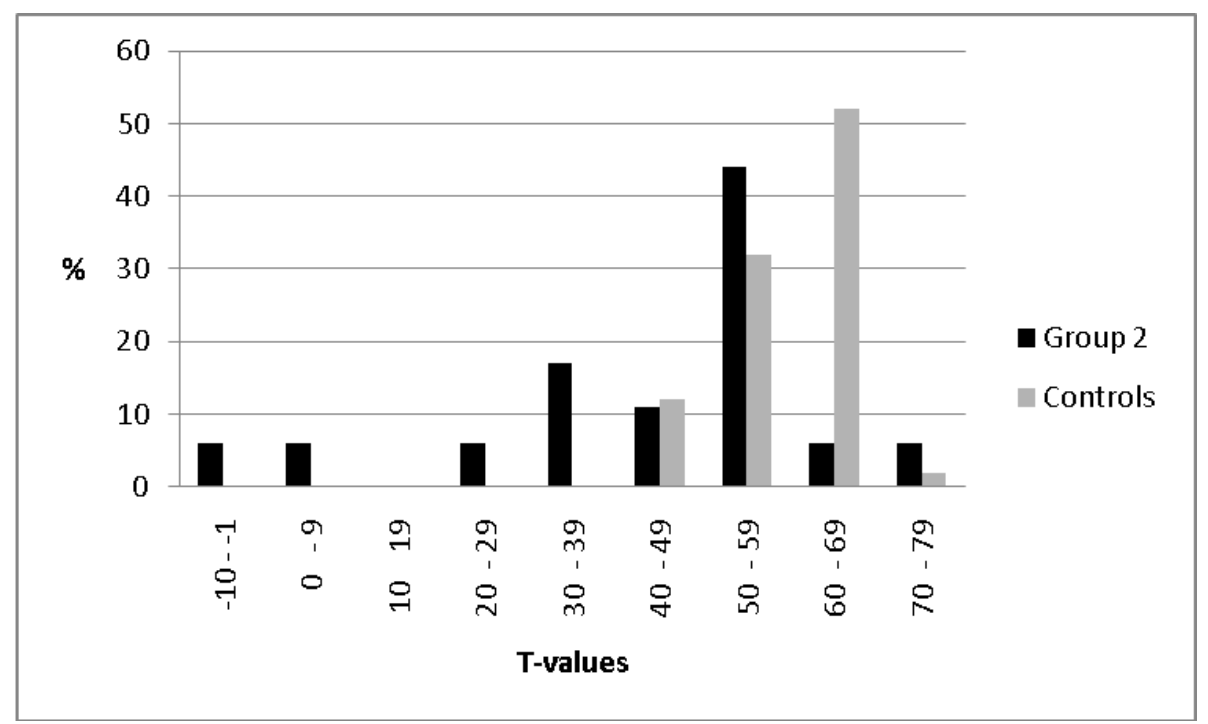

FIGURE 11. Individual outcomes of Study 3 in the domain of verbal memory for Group 2 and the control group

One way to evaluate different paths of development is to study subgroups based on moderators such as the age at the time of injury, pre-injury factors concerning the family and the child, injury-related factors, treatment-related factors, post-injury factors including family function, rehabilitation, time since the injury and age at the time of the injury. Research involving subgroups of patients with a CTBI can be challenging, though. Since cohorts of clinical paediatric groups often are small and heterogeneous, the statistical analysis gets complicated (Smedler \& Winiarski, 2008). Further division into subgroups based on different moderators diminishes the groups even more, further diminishing statistical power.

Another way of investigating different paths of development is chosen by Fay et al. (2009). They used a person-oriented approach when looking at longitudinal patterns of functional deficits after a CTBI. This made it possible to study different courses of recovery of the individuals within the severe group. There was variation concerning the deficits within the group of severely injured patients 4 years post-injury, with some having persistent deficits in all of the four outcome domains in the study (neuropsychological, academic, adaptive and behaviour) while others did not have deficits in one or more outcome domains, a variation that would not have shown up if the result had been presented with mean values from a variable oriented, group based design.

In the investigation of the relationship between brain-injury parameters and early functional outcome in Study 2, we used a person-oriented approach. We investigated the clusters of individual brain injury parameters, 
which provides information about the profile of the parameters, as they occur for the individuals. This approach paves the way for a holistic evaluation, relying on the theoretical assumption that the parameters can have a dynamic relationship that holds important information (Bergman, Magnusson \& El-Khouri, 2009), in this case, information concerning how early parameters taken together may predict early functional outcome. Bergman et al. (2009) argue that individual development is best understood from the perspective of processes and from the use of holistic-interactionistic, integrated models, since development often has a non-linear and dynamic character. A person-oriented holistic approach might be helpful in answering a question Beaulieu (2002) claims to be important for augmenting the availability of rehabilitation: what does a good candidate for rehabilitation look like? According to the individual variation within the groups of those with a CTBI, that emerges as an important question for further research.

\section{Summary of new knowledge}

The new knowledge added by the research presented in this thesis is summarised below and then discussed in relation to the opening model of the thesis, described in Figure 1. The contributions of the thesis to the model are summarised if Figure 12, page 56.

- There are significant differences between the former groups of patients neurosurgically treated for CTBI, 6-13 years after they suffered the injury compared with non-injured controls.

- Long-term cognitive deficits of a similar magnitude and character, significantly lower than those of uninjured groups where found in the two cohorts, treated before and after advancements in the neurosurgical care.

- In long-term outcome 6-13 after CTBI, verbal intellectual functions and executive functions were found to be vulnerable while visuospatial functions appeared to have a better long-term recovery.

- Two Swedish cohorts, neurosurgically treated for a CTBI before and after advancements in neurosurgical care, had a similar rate of survival and an equally low rate of admittance to rehabilitation.

- An exploratory evaluation of the relationship between early brain injury parameters and early functional outcome indicates that the length of time in intensive care and the length of care in a respirator may have a stronger relationship to early outcome than does a single measure of the level of consciousness at admission. The result provides an example of how a person-oriented holistic approach can be used to evaluate profiles of predictors of outcome. 
This thesis has shown that there are significant differences between the patients neurosurgically treated for CTBI, 6-13 years after they suffered the injury compared with non-injured controls. The outcome of children with CTBI, neurosurgically treated, have previously not been evaluated after such an extensive period of time. Studies 1 and 3 are based on a small sample and need to be replicated. Despite this, it should be noted that the groups involved in studies of CTBI are generally small and are also heterogeneous, implying the necessity to communicate results obtained from several studies to develop a critical knowledgebase.

Verbal intellectual functions and executive functions were found to be vulnerable for long-term cognitive outcome, while visuospatial functions appeared to have a better long-term recovery. The result in this thesis support the finding that visuospatial intellectual functions recover better than verbal intellectual or executive functions (Babikian \& Asnarow, 2009), but we add the knowledge that this is also the case at a follow-up as long as 6-13 years after the injury. We suggest that it implies better development of visuospatial functions after a CTBI, however this has to be evaluated in further studies. In contrast, the low results for verbal intellectual, verbal memory and executive functions indicate that these either have a more limited recovery, or that the development of those functions is hampered. In this thesis, the low verbal IQ and verbal memory function are interpreted as being the result of hampered development of verbal functions, and of hampered development of the executive control over verbal functions. These results provide new knowledge about Cognitive long-term outcome, particularly in relation to Time since injury and also Present age. The indication of a better recovery of visuospatial functions provides hypotheses regarding Plasticity and Recovery of functions.

Our results have also contributed by providing new data on Swedish patients, together with a recent study based partly on the same sample. Horneman \& Emanuelson (2009) reported the long-term outcome of the complete group of 53 people treated in the earlier period who had been subject to the neuropsychological follow-up assessment presented in Figure 2, page 32. This thesis adds knowledge about the specific group of neurosurgically treated patients, treated before (Group 1) and after (Group 2) advancements in neurosurgical care had taken place. It also presents the results of longterm cognitive deficits of a similar magnitude and character in both groups, and shows the cognitive functions of those concerned to be significantly lower than those of uninjured groups. These results provides new knowledge of the moderating influence of Initial CTBI treatment.

The evaluation of the relationship between early brain injury parameters and early functional outcome indicates that the length of time in intensive care and the length of care in a respirator may have a stronger relationship to outcome than does a single measure of the level of consciousness. The result 
is explorative but provides an example of how a person-oriented holistic approach can be used to evaluate profiles of predictors of outcome.

Limited information is found regarding Coping with disabilities, more precisely the environmental coping. Study 1 described the process of initial information and the following school- and work adjustments, with the discouraging results of initial information of cognitive deficits for five of the eight persons being followed by no school adjustments, and that three of the eight persons went from a school situation with no adjustments to adult life with an early retirement.

We tried to evaluate the moderating effect of Gender, but subgroups became too small for statistical power. The same state was true for Age at injury.

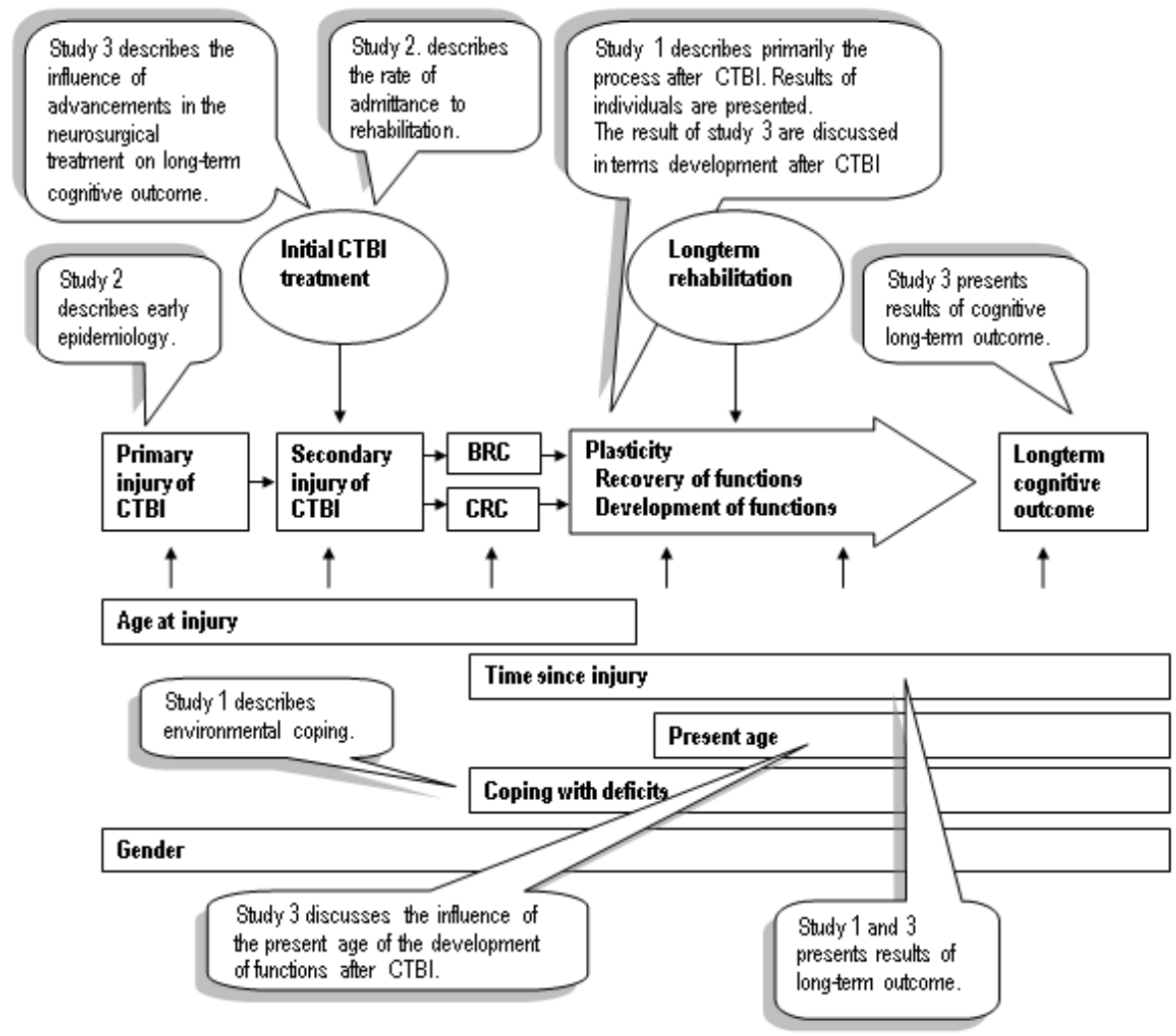

FIGURE 12. Influences on long-term cognitive outcome after a CTBI and the contribution to the model from the research in this thesis 


\section{Limitations}

The number of subjects was very small, particularly in Study 1. The first assessment was made on a clinical basis and was not based on a standardised test battery, as in assessments two and three. Many of the tests in the first assessment differed from the ones in the following assessments, thus reducing the number of tests available for the purpose of this study. The measures used in the article were chosen because they offered the possibility of making reliable and valid comparisons over the three occasions, but despite this, the conditions for comparison were not optimal.

In Study 3, the original groups were limited in size, as is often the case in clinical studies. Furthermore, cognitive results were only available for $30 \%$ and 39\% of the original surviving patients. However, there were no systematic differences between the study-groups and their original groups, indicating that the study-groups did hold representativity of the original groups. However, the sample sizes are small and the results of this study need to be replicated in further studies, with larger samples, preferably obtained through multi-centre collaboration.

In Study 3, there was a difference between the groups concerning the amount of time to have elapsed since injury: 13.2 years for Group 1 and 6.1 years for Group 2. The most extensive changes in the development after CTBI occur in the year immediately subsequent to the injury (Chadwick, Rutter, Brown, Shaffer \& Traub, 1981; Ewing-Cobbs et al., 1997; Yeates et al., 2002). However, as both groups were far beyond the first year after injury, aspects of recovery unique to the first period, should not affect the groups differently. Concerning the long-term development, verbal and executive functions appear to be central. Since the patients in Group 2, with a mean age of 17, appear to be going through the developmental period with limited brain capacity, it is possible that their verbal intellectual deficits might become more pronounced over time.

The small sample in study 1 was also part of Group 1 in Study 3, consisting of some of the most injured persons in that group. However, the longitudinal assessments in Study 1 allowed for a developmental analysis, which complements the result from a single assessment that was the base in Study 3. Still, one should keep in mind that the long-term deficits in verbal and executive functions reported in both studies emanate from overlapping samples and that these findings need to cross-validated.

The somewhat surprising profile of the control group in Study 3 is commented on page 47, in the section "General discussion". A possible explanation presented was that the norms of the subtests come from different populations, and therefore the profiles of the cognitive performance should not be interpreted. In Study 1, the result is based on trajectory profile analyses even though the norms of some of the results in the same domain originate from 
different subtests and populations. We aimed to minimise this bias by choosing tests with measures of acceptable validity.

In Study 2 we regarded Group 2 as a population in the evaluation of relationship between early brain injury parameters and early functional outcome. Following that, the result describes the situation for the actual population, but the study is small and exploratory and the findings cannot be generalised to other samples or populations. The result has to be replicated in larger groups.

\section{Suggestions for further research}

According to the literature of long-term development after a CTBI, it is apparent that we have but limited knowledge about how brain injury affects and interacts with the child's ongoing development. The literature provides results on outcome measures, but the understanding is limited about plasticity after CTBI, in terms of the brain-behaviour-environmental influences on recovery and development. We know that CTBI poses a threat to functional development, but there are important questions for future research.

The results presented in this thesis suggest that adolescent development of verbal intellectual and executive functions may broaden the gap between groups with CTBI and controls. This needs to be further elucidated in longitudinal studies.

Age at injury has been presented as the time-variable most frequently examined. Present age, or age at testing, is a time-variable more rarely examined. Adding the moderator of present age, a longitudinal, person-oriented approach could provide knowledge about the ongoing development after CTBI, at different post-injury intervals and different developmental stages. Such an approach could investigate the long-term interplay between recovery and development influencing the outcome of verbal memory after adolescence. A holistic person-oriented approach might also be helpful in evaluating how the relation of the moderating factors, described in Figure 1, influence the long-term outcome of a CTBI on the individual level. The same method could also be used to identify profiles of predictors of long-term outcome.

There is also a great need for intervention studies in this field: What promotes positive development after a CTBI, with regard to the interplay of age at injury, present age and time since injury? With such knowledge, what kind of rehabilitation interventions would be most beneficial at different ages? This knowledge is almost non existing in the literature today.

As new treatment protocols are introduced in intensive neruosurgical care, there is a continuous need for long-term follow-up studies. Ideally, patients with comparable brain injuries but treated according to different protocols should be matched and compared on relevant outcome measures. In reality, 
such a quasi-experimental design is almost impossible to achieve. However, multi-centre efforts and international collaboration may bring us closer to achieving such a knowledge base.

\section{Conclusions}

Deficits in the cognitive domains of verbal and visuospatial intellectual functions, executive functions and memory functions are apparent at long-term follow up, 6-13 years after neurosurgically treated CTBI, even after advancements in the neurosurgical care in Sweden. The time since the insult is an important factor when assessing the outcome after a TBI in childhood and adolescence, and therefore the assessment of final outcome should not be conducted before the child reaches adulthood.

Measures of verbal IQ, verbal memory and executive functions were especially low at long-term cognitive follow-up. Verbal learning and the executive control of memory functions should be addressed with interventions aimed at coping and compensation. Visuospatial intellectual functions, in contrast, stand out as having a better long-term recovery.

Despite important advancements in the neurosurgical treatment after a CTBI, and despite an increasing amount of reports being published of cognitive and behavioural deficits after a CTBI, the current arrangements for a referral to rehabilitation and follow-up for the children concerned have not improved and are still insufficient in Sweden.

The scientific knowledgebase is meagre where the long-term cognitive developmental trajectories of recovery and development after CTBI are concerned. There is also a limited knowledge of the interaction between how the moderators of cognitive outcome interact. A person-oriented holistic approach can be used to evaluate the profiles of predictors of individual outcome. 


\section{Acknowledgements}

A thesis is not made by a single person.

My primary gratefulness goes to my supervisors. Associate Professor AnnCharlotte Smedler has with a clear eye and trustworthy attendance guided me through details and overview of scientific methodology in the field of child neuropsychology, strengthening my writing to a level I had not reached on my own. Thank You!

Associate Professor Ingrid Emanuelson welcomed me into the area of research and generously gave me the opportunity of joining projects where the first fundamental parts were already done. She has supported me all the way through the area of TBI, which she often knows as her own back yard. Thank You!

When I for the first time, 2002, raised the question of whether there was a research project on CTBI in Sweden that I could join - Göran Horneman, neuropsychologist and $\mathrm{PhD}$, said yes. I am also in gratitude and sorrow of Mia Leis Ljungmark, co-author in the third article, who unexpectedly passed away during our work. Professor Bertil Rydenhag gave valuable contributions to Study 2 and 3, and Associate Professor Hans Silander and AnnaKarin Åkerman, physician, contributed to Study 2. Thank You!

As external readers, Professor Ove Almkvist and Professor Annika Dahlgren Sandberg contributed with constructive feed-back on the thesis. Professor Lars Bergman gave valuable support in questions about person-oriented methodology. Suzanne Lidström did a tremendous job in changing the language from Swenglish to British English. Thank You!

The Department of Psychology, Stockholm University, accepted me as a doctoral student and gave financial and practical support through these years, even though I as a long-distance student did have very limited possibilities to contribute to the activities at the department. This secured the supervision in my specific area of psychology, which was not possible in my hometown. Thank You! 
Without support from my home organization, Jämtland County Council, the thesis would not have been possible to carry through. At the Research and Development Unit, Ragnar Asplund was the director when my application of a doctoral training position was accepted. Christina Reuterwall took the following leadership, giving me valuable support in the research process. Susanne Johansson has helped me keeping track of all the administrative details, and also guidance in questions about SPSS. Thank You!

The Child and Youth Rehabilitation, Östersund Hospital, has since long reached for a high level of knowledge in the work with children with disabilities. The manager of that time, Kia Carlsson also gave positive support throughout the research process. The clinical work of rehabilitation after acquired brain injuries has been the core of the motivation for this work. Since we in the middle of 1990 started to organise how we could meet and work with children and families in this complicated and serious area, important co-workers among others have been: Urban Eriksson, Elsine Lien, Ulla Stahre Påhlsson, Sara Halvarsson, Eva Nyström Cederberg, Ingela Kristianssen, Agnetha Borell, Janolov Johnsson, Anna Blomgren, Carina Stenström and Gunilla Holm Persson. Thank You!

At Östersund Hopspital, the Hospital Library and Helpdesk, especially Gun Lind, have given important guidance at innumerable occasions. Thank You!

The work with this thesis has also involved my family. Roger, you stood by my side and encouraged me all the way. Axel and Clara, you accepted to take more of the work in the kitchen when I was away, on the road between Stockholm, Gothenburg and Östersund. Thank You!

My mother Margareta has been my back-up in the search of the right English prepositions throughout these years, doing the language check-up in the Study 1, and finally the last language check-up of the thesis. Thank You!

Catherine Aaro Jonsson

Frösön, 27 april 2010 


\section{References}

Anderson, V. (2007) Childhood White Matter Injuries: What Are The Issues? Developmental Neuropsychology 32, 619-623.

Anderson, V., Anderson, P., Northam, E., Jacobs, R. \& Catroppa, C. (2001a) Development of Executive Functions Through Late Childhood and Adolescence in an Australian Sample. Developmental Neuropsychology 20, 385-406.

Anderson, V., Brown, S., Newitt, H., Hoile, H. (2009a) Educational, Vocational, Psychosocial, and Quality-of-Life Outcomes for Adult Survivors of Childhood Traumatic Brain Injury. Journal of Head Trauma Rehabilitation 24, 303-312.

Anderson, V. \& Catroppa, C. (2006) Advances in Postacute Rehabilitation after Childhood Acquired Brain Injury. American Journal of Physical Medicine and Rehabilitation 85, 767-778.

Anderson, V., Catroppa, C. (2007) Memory outcome at 5 years postchildhood traumatic brain injury. Brain Injury 21,1399-1409.

Anderson, V., Catroppa, C., Dudgeon, P., Morse, S. A., Haritou, F. \& Rosenfeld, J. V. (2006). Understanding Predictors of functional recovery and outcome 30 months following early childhood head injury. Neuropsychology 20, 42-57.

Anderson, V., Catroppa, C., Haritou, F, Morse, S., Pentland, L., Rosenfeld, J. \& Stargatt, R. (2001b) Predictors of Acute Child and Family Outcome following Traumatic Brain Injury in Children. Pediatric Neurosurgy 34, $138-148$.

Anderson, V., Catroppa, C., Morse, S., Haritou, F. \& Rosenfeld, J. (2000) Recovery of Intellectual Ability following Traumatic Brain Injury Severity and Age at Injury. Pediatric Neurosurgery 32: 282-290.

Anderson, V., Catroppa, C., Morse, S., Haritou, F. \& Rosenfeld, J. (2009b) Intellectual outcome from preschool traumatic brain injury: a 5-year prospective, longitudinal study. Pediatrics 124, 1064-1071.

Anderson, V., Catroppa, C., Morse, S., Haritou, F. \& Rosenfeld, J. (2005) Functional plasticity or vulnerability after early brain injury? Pediatrics 116, 1374-1382. 
Anderson, V., Fenwick, T., Manly, T. \& Robertson I. (1998) Attentional skills following traumatic brain injury in childhood: a componential analysis. Brain Injury 12, 937-949.

Anderson. V., Morse, S., Catroppa, C., Haritou, F. \& Rosenfeld, J.V. (2004) Thirty month outcome from early childhood head injury: a prospective analysis of neurobehavioural recovery. Brain 127, 2608-2620.

Anderson, V., Spencer-Smith, M., Leventer, R., Coleman, L., Anderson, P., Williams, J...Jacobs, R. (2009c) Childhood brain insult: can age at insult help us predict outcome? Brain 132, 45-56.

Archinegas, D., Adler, L., Topkoff, J., Cawthra, E., Filley, C. M \& Reite, M. (1999) Attention and memory dysfunction after traumatic brain injury: cholinergic mechanisms, sensory gating, and a hypothesis for further investigation. Brain Injury 13, 1-13.

Asgeirsson, B., Grande, P.O. \& Nordstrom, C.H. (1994) A new therapy of post-trauma brain oedema based on haemodynamic principles for brain volume regulation. Intensive Care Medicine 20, 260-267.

Babikian, T. \& Asnarow, R. (2009) Neurocognitive Outcomes and Recovery After Pediatric TBI: Meta-Analytic review of the Literature Neuropsychology 23, 283-296.

Beardmore, S., Tate, R. \& Liddle, B. (1999) Does Information and Feedback Improve Children's Knowledge and Awareness of Deficits after Traumatic Brain Injury? Neuropsychological Rehabilitation 9, 45-62.

Beaulieu, C. L. (2002) Rehabilitation and outcome following pediatric traumatic brain injury. Surgical Clinics of North America 82, 393-408.

Bell, M. A., Fox, N. A. (1994) Brain Development over the First Year of Life. In G. Dawson \& K. W. Fisher (Eds), Human Behavior and the Developing Brain (pp 314-345). New York: The Guilford Press.

Bergman, L. R., Magnusson, D., El-Khouri, B., M. (2003) Studying individual development in an interindividual context: A person oriented approach. New York, NY: Psychology Press.

Birkenkamp, R., \& Zillmer, E. (1998) The d2 Test of Attention. Goettingen: Hogrefe.

Boll, T. (1993) Children's Category test. San Antonio, TX: The Psychological Corporation.

Bornstein, R A. (1985) Normative data on selected neuropsychological measures from a nonclinical sample. Journal of Clinical Psychology 41, 651-659. 
Braga, L.W., Da Paz Junior, A.C. \& Ylvisaker, M. (2005) Direct cliniciandelivered versus indirect family-supported rehabilitation of children with traumatic brain injury: A randomized controlled trial. Brain Injury 19, 819-831.

Butler, R. W. \& Copeland, D, R. (2002) Attentional processes and their remediation in children treated for cancer: A literature review and the development of a therapeutic approach. Journal of the International Neuropsychological Society 8, 115-124.

Böhm, B., Lundequist, A. \& Smedler, A-C. (2010) Visual-motor and executive functions in children born preterm: the Bender Visual Motor Gestalt revisited. Scandinavian Journal of Psychology, in press.

Carlsson, G. (1994) Children with hemiplegic cerebral palsy: neuropsychological consequences of early unilateral brain lesions (Doctoral dissertation, University of Gothenburg, 1994). ISBN 91-628-1396-X.

Casey, B., Giedd, J. \& Thomas, K. (2000) Structural and functional brain development and its relation to cognitive development. Biological Psychology 54, 241-257.

Catroppa, C. \& Anderson, V. (2002) Recovery in memory function in the first year following TBI in children. Brain Injury 16, 369-384.

Catroppa, C., Anderson, V. (2007) Recovery in memory function, and its relationship to academic success, at 24 months following pediatric TBI. Child Neuropsychology 13, 240-261.

Catroppa, C. \& Anderson, V. (2008) Outcome and Predictors of Functional Recovery 5 Years Following Pediatric Traumatic Brain Injury (TBI). Journal of Pediatric Psychology 33, 707-718.

Catroppe, C. \& Anderson, V. (2009) Traumatic brain injury in childhood: Rehabilitation considerations. Developmental Neurorehabilitation 12, 53-61.

Catroppa, C., Anderson, V., Ditchfield, M., Coleman, L. (2008) Using magnetic resonance imaging to predict new learning outcome at 5 years after childhood traumatic brain injury. Journal of Child Neurology 23, 486496.

Cattelani, R., Lombardi, F., Brianti, R. \& Mazzucchi, A. (1998) Traumatic brain injury in childhood: Intellectual, behavioural and social outcome into adulthood. Brain Injury 12, 283-296.

Chadwick, O., Rutter, M., Brown, G., Shaffer, D. \& Traub, M.U. (1981a) A prospective study of children with head injuries: II, Cognitive sequelae. Psychological Medicine 11, 49-61. 
Chadwick, O., Rutter, M., Shaffer, D., Shrout, P. E. (1981b) A prospective study of children with head injuries: IV, Specific Cognitive Deficits. Journal of Cognitive Neuropsychology 3, 101-120.

Chapman, S. B., McKinnon, L., Levon, H. S., Song, J., Meier, M. C., Chiu, S. (2001) Longitudinal Outcome of Verbal Discourse in Children with Traumatic Brain Injury: Three-Year Follow-Up. Journal of Head Trauma Rehabilitation 16, 441- 455.

Christensen, A-L. (1984) Luria's neuropsychological investigation. 2nd ed. Copenhagen: Munksgaard.

Copeland, D. R. \& Fletcher, J.M. (1985) Neuropsychological Sequelae in Childhood Cancer in Long-Term Survivors. Pediatrics 75, 745-753.

Crosson, B., Barco, P.P., Velozo, C.A., Bolesta, M.M., Cooper, P.V. \& Werts, D.W. (1989) Awareness and compensation in postacute head injury rehabilitation. Journal of Head Trauma Rehabilitation 4, 46-54.

De Luca, C., Leventer, R., J. (2008) Developmental trajectories of executive functions across the lifespan. In v. Anderson, R Jacobs \& P., J. Anderson (Eds) Executive functions and the frontal lobes. A lifespan Perspective. (pp. 24-56) New York, London: Taylor \& Francis.

De Renzi, E. \& Faglioni, P. (1978) Normative data and screening power of a shortened version of the Token Test. Cortex 14, 41-49.

Dennis, M., Guger, S., Roncadin, C., Barnes, M. \& Schachar, R. (2001) Attentional-inhibitory control and social-behavioral regulation after childhood closed head injury: Do biological, developmental, and recovery variables predict outcome? Journal of the International Neuropsychological Society 7, 683-692.

Dennis, M., Yeates, K. O., Taylor, H. G. \& Fletcher, J. M. (2007) Brain reserve capacity, cognitive reserve capacity, and age-based functional plasticity after congenital and acquired brain injury in children. In Y Stern (Ed.), Cognitive reserve. Theory and applications (pp. 53-83). New York and London: Taylor and Frances.

Donders, J. \& Giroux, A. (2005) Discrepancies between the California Verbal Learning Test: Children's version and the Children's Category Test after pediatric traumatic brain injury. Journal of the International Neuropsychological Society 11, 386-391.

Donders, J. \& Hoffman, N., M. (2000) Gender differences in learning and memory after pediatric traumatic brain injury. Neuropsychology 16, 491499.

Donders, J. \& Warschausky, S. (2007) Neurobehavioral Outcomes After Early Versus Late Childhood Traumatic Brain Injury. Journal of Head Trauma Rehabilitation 22, 296-302. 
Donders, J., Woodward, H. (2003) Gender as a moderator of memory after traumatic brain injury in children. Journal of Head Trauma Rehabilitation 18, 106-115.

Dunn, L.M., Dunn, L.M. (1981) Peabody Picture Vocabulary Test - Revised. Circle Pines, MN: American Guidance Service.

Eker, C., Schalén, W., Asgeirsson, B., Grände, P-O., Ranstam, J. \& Nordström, C-H. (2000) Reduced mortality after severe head injury will increase the demands for rehabilitation services. Brain Injury 14, 605-619.

Emanuelson, I. (1999) Epidemiology and management of traumatic brain injury in children (Doctoral dissertation, University of Helsinki, 1999) ISBN 91-628-3445-2.

Emanuelson, I., von Wendt, L. (1997) Epidemiology of traumatic brain injury in children and adolescents in south-western Sweden. Acta Paediatrica 86, 730-735.

Emanuelson, I., von Wendt, L., Lundälv, E. \& Larsson, J. (1996) Rehabilitation and follow-up of children with severe traumatic brain injury. Child's Nervous System 12, 460-465.

Emanuelson, I., von Went, L., Beckung, E. \& Hagberg, I. (1998) Late outcome after severe traumatic brain injury in children and adolescents. $\mathrm{Pe}$ diatric Rehabilitation 65, 70-72.

Engman, M-L., Adolfsson, I., Lewensohn-Fuchs, I., Forsgren, M., Mosskin, M., Malm, G. (2008) Neuropsychologic Outcomes in Children With Neonatal Herpes Encephalitis. Pediatric Neurology 38, 398-405.

Enlund, M., Mentell, O., Engström, C., Horneman, G., Rönquist, G. (1996) Occurrence of Adenalyte Kinase in Cerebrospinal Fluid after Isoflurance Anaesthesia and Orthognatic Surgery. Upsala Journal of Medical Sciences 101, 97-111.

Ewing-Cobbs, L., Fletcher, J, M., Levin, H, S., Francis, D. J., Davidson, K. \& Miner, M. E. (1997) Longitudinal neuropsychological outcome in infants and preeschoolers with traumatic brain injury. Journal of the International Neuropsychological Society 3, 581-591.

Fay, T.B., Yeates, K. O., Taylor, H. G., Bangert, B., Dietrich, A., Nuss, K. E.,...Wright, M. (2010) Cognitive reserve as a moderator of postconcussive symptoms in children with complicated and uncomplicated mild traumatic brain injury. Journal of the International Neuropsychological Society 16, 94-105.

Fay, T.B., Yeates, K.O., Wade, S., Drotar, D. \& Stacin, T. (2009) Predicting Longitudinal Patterns of Functional Deficits in Children With Traumatic Brain Injury. Neuropsychology 23, 271-282. 
Finlayson, M. A., \& Reitan, R M. (1976) Handedness in relation to measures of motor and tactile perceptual function in normal children. Perceptual and Motor Skills 43, 475-481.

Forrester, G. \& Geffen, G. (1991) Performance measure of 7 to 15 year old children on the Auditory Verbal Learning Test. The Clinical Neuropsychologist 5, 345-359.

Frith, C. D., \& Frith, U. (2007) Social Cognition in Humans. Current Biology 17, R724-R732.

Gaddes, W. H., \& Crockett, D. J. (1975) The Spreen-Benton aphasia test: Normative data as a measure of normal language development. Brain and Language 2, 257-280.

Geffen, G., Hoar, K. J., Ó Hanlon, A.P., Clarc, C. R. \& Geffen, L. B. (1990) Performance measures of 16- to 86-year old males and females on the auditory Verbal Learning Test. The Clinical Neuropsychologist 4, 45-63.

Gogtay, N., Giedd, J. N., Lusk, L., Hayashi, K.M., Greenstein, D., Vaituzis, C.,... Thompson, P.M. (2004) Dynamic mapping of human cortical development during childhood through early childhood. Proceedings of the National Academy of Sciences of the United States of America 101, 8174-8179.

Grande, P.O. (1989) The effects of dihydroergotamine in patients with head injury and raised intracranial pressure. Intensive Care Medicine 15, 523527.

Grande, P.O. (2006) The "Lund Concept" for the treatment of severe head trauma--physiological principles and clinical application. Intensive Care Medicine 32, 1475-1484.

Green, C., R., Mihik, S. M., Nikkel, B., C., Stade, B., C., Rasmussen, C., Munoz, D., P.,...Reynolds, J., N. (2009) Executive function deficits in children with fetalalcohol spectrum disorders (FASD) measured using the Cambridge Neuropsychological Tests Automated Battery (CANTAB). Journal of child psychology and psychiatry 50, 688-697.

Groswasser, Z., Cohen, M. \& Keren, O. (1998) Female TBI patients recover better than males. Brain Injury 12, 805-808.

Hanten, G., Dennis, M., Zhang, L., Barnes, M., Roberson, G., Archibald, J.,...Levin, H.S. (2004) Childhood Head Injury and Metacognitive Processes in Language and Memory. Developmental Neuropsychology 25, 85-106.

Havnesköld, L. \& Risholm Mothander, P. (2009) Utvecklingspsykologi. Stockholm: Liber. 
Hawley, C.A. (2003) Reported problems and their resolution following mild, moderate and severe traumatic brain injury among children and adolescents in the UK. Brain Injury 17, 105-129.

Hebb, D. O. (1942) The effect of early and late brain injury upon test scores, and the nature of normal adult intelligence. Proceedings of the American Philosophical Society 85, 275-292.

Horneman, G. \& Emanuelson, I. (2009) Cognitive outcome in children and young adults who sustained severe and moderate traumatic brain injury 10 years earlier. Brain Injury 23, 907-914.

Horneman, G., Folkesson, P., Sintonen, H., von Wendt, L. \& Emanuelson, I. (2005) Health related quality of life of adolescents and young adults 10 years after serious traumatic brain injury. International Journal of Rehabilitation Research 28, 245-249.

Jacobs, R., Harvey, A., S. \& Anderson, V. (2007) Executive control following focal frontal lobe lesions: impact of timing of lesions on outcome. Cortex 43, 792-805.

Jennett, B. \& Bond, M. (1975) Assessment of outcome after severe brain damage. Lancet 1, 480-484.

Johnston, M. V. (2009) Plasticity in the Developing Brain: Implications for rehabilitation. Developmental Disabilities Research Reviews 15, 94-101.

Johnstone, A. J., Lohlun, J. C., Miller, J. D., McIntosh, C. A., Gregori, A., Brown, R.,...Tocher, J. L. (1993) A comparison of the Glasgow Coma Scale and the Swedish Reaction Level Scale. Brain Injury 7, 501-506.

Keenan, H. T. \& Bratton, S. L. (2006) Epidemiology and Outcomes of Pediatric Traumatic Brain Injury. Developmental Neuroscience 28, 256-263.

Kennard, M. (1936) Age and other factors in motor recovery from precentral lesions in monkeys. American Journal of Physiology 115, 138-46.

Klonoff, H.,Clarc, C. \& Klonoff, P,S. (1993) Long-term outcome of head injuries: A 23 year follow-up study of children with head injuries. Journal of Neurology and Neurosurgical Psychiatry 56, 410-415.

Kochanek, P. M. (2006) Pediatric Traumatic Brain Injury: Quo Vadis? Developmental Neuroscience 28, 244-255.

Kolb, B., Pellis, S. \& Robinson, T. E. (2004) Plasticity and functions of the orbital frontal cortex. Brain and Cognition 55, 104-115.

Koskiniemi, M., Kykkä, T., Nybo, T. \& Jarho, L. (1995) Long-term outcome after severe brain injury in pree-schoolers is worse than expected. Archives of Pediatrics \& Adolescent Medicine 149, 449-454. 
Kramer, M.E., Chiu, C-Y. P., Walz, N. C., Holland, S. K., Yuan, W., Karunanayaka, P. \& Wade, S. L. (2008) Long-term neural processing following early childhood traumatic brain injury: fMRI and neurobehavioral outcomes. Journal of the International Neuropsychological Society 14, 424-435.

Kraus, J.F. (1995) Epidemiological features of brain injury in children: occurrence, children at risk, causes and manner of injury, severity and outcomes. In: Broman, S.H. \& Michel, M.E. eds. Traumatic head injury in children. (pp. 22-37) New York: Oxford University Press.

Laatsch, L., Harrington, D., Hotz, G., Marcantuono J., Mozzoni, M P., Walsh V. \& Pike Hersey K. (2007) An evidence-based Review of Cognitive and Behavioral Rehabilitation Treatment Studies in Children With Acquired Brain Injury. Journal of Head Trauma Rehabilitation 22, 248256.

Lehnung, M., Leplow, B., Ekroll, Benz, B., Ritz, A., Mehdorn, M. \& Ferstl, R. (2003) Recovery of spatial memory and persistence of spatial orientation deficits after traumatic brain injury during childhood. Brain Injury 17, 855-869.

Lehnung, M., Leplow, B., Herzog, A., Benz B., Ritz, A., Stolze, H.,...Ferstl, R. (2001). Children's Spatial Behaviour is Differentially Affected After Traumatic Brain Injury. Child Neuropsychology 7, 59-71.

Levin, H. S. (2003) Neuroplasticity following non-penetrating traumatic brain injury. Brain Injury 17, 665-674.

Levin, H. S. \& Hanten, G. (2005) Executive functions after traumatic brain injury in children. Pediatric Neurology 33, 79-93.

Levin, H., Hanten, G., Max, J., Li, X., Swank, P., Ewing-Cobbs, L..,. Schachar, R. (2007) Symptoms of Attention-Deficit/Hyperactivity Disorder Following Traumatic Brain Injury in Children. Journal of Developmental \& Behavioral Pediatrics 28, 108-118.

Levin, H. S., Song, J., Ewing-Cobbs, L., Chapman, S. \% Mendelsohn, D. (2001) Word fluency in relation to severity of closed head injury, associated frontal brain lesions, and age at children. Neuropsychologia 39, $122-13$.

Limond, J., Leeke, R. (2009) Practioner review: Cognitive rehabilitation for children with acquired brain injury. Journal of Child Psychology and Psychiatry 46, 339-352.

Massagli, T. L., Michaud, L. J., Rivara, F. P. (1996) Association between injury indices and outcome after severe brain injury in children. Archives of Physical Medicine and Rehabilitation 77, 125-32. 
McDougall, J., Servais, M., Sommerfreund, J., Rosen, E., Gillett, J., Grey, J.,..Hicock, F. (2006) An evaluation of the paediatric acquired brain injury community outreach programme (PABICOP). Brain Injury 20, 1189-1205.

Mendelsohn, D., Levin, H. S., Bruce, D., Lilly, M., Harward, H., Culhane, K. A., Eisenberg, H. M. (1992) Late MRI after head injury in children: relationship to clinical features and outcome. Child's Nervous System: official journal of the International Society for Pediatric Neurosurgery $8,445-452$.

Meyers J E. \& Meyers K R. (1995) Rey Complex Figure Test and Recognition Trail: Professional manual. Odessa, FL: Psychological Assessment resources.

Michaud, L. J., Rivara, F. P., Grady, M. S., Reay, D. T. (1992) Predictors of survival and severity of disability after severe brain injury in children. Neurosurgery 31, 254-64.

Muscara, F., Catroppa, C. \& Anderson, V. (2008) The impact of injury severity on executive function 7-10 years following pediatric traumatic brain injury. Developmental Neuropsychology 33, 623-636.

Nagy, Z., Ashburner, J., Andersson,J., Jbabdi, S., Draganski, B., Skare, S.,.. Lagercrantz, H. (2009) Structural Correlates of Preterm Birth in the Adolescent Brain. Pediatrics 124, 964-972.

Niedzwecki, C. M., Marwitz, J. H., Ketchum, J. M., Cifu, D. X., Dillard, C. M., Monasterio, E. A. (2008) Traumatic brain injury: a comparison of inpatient functional outcomes between children and adults. Journal of Head Trauma Rehabilitation 23, 209-219.

Niemeier, J. P., Marwitz, J. H., Lesher, K., Walker, W.C. (2007) Gender differences in executive functions following traumatic brain injury. Neuropsychological Rehabilitation 17, 293-313.

Nybo, T., Sainio, M. \& Muller, K. (2004) Stability of vocational outcome in adulthood after moderate to severe preschool brain injury. Journal of the International Neuropsychological Society 10, 719-723.

Parslow, R.C., Morris, K.P., Tasker, R.C., Forsyth, R.J. \& Hawley, C.A. (2005) Epidemiology of brain injury in children receiving intensive care in the UK. Archives in Disease in Childhood 90, 1182-1187.

Piaget, J. (1982) Barnets själsliga utveckling. Lund, Swe: Liber Förlag.

Ponsford, J., Willmott, C., Rothwell, A., Cameron, P., Ayton, G., Nelms, R.,... Ng, K. (2001) Impact of Early Interbentions on Outcome After Mild Traumatic Brain Injury in Children. Pediatrics 108, 1297-1303. 
Prigatano, G.P. \& Grey, J. (2008) Parental Perspectives on Recovery and Social Reintegration After Pediatric Traumatic Brain Injury. Journal of Head Trauma Rehabilitation 23, 378-387.

Ratcliff, J. J., Greenspan, A., Goldstein, F. Stringer, A.Y., Bushnik, T., Hammonds, F.M.,...Wright, D.W. (2006) Gender and traumatic brain injury: Do the sexes fare differently? Brain Injury 2, 1023-1030.

Rogers, E., Wagner, A. K. (2006) Gender, sex steroids, and neuroprotection following traumatic brain injury. Journal of Head Trauma Rehabilitation 21, 279-281.

Roncadin, C., Pascual-Leone, J., Rich, J. B. \& Dennis, M. (2007) Developmental relations between working memory and inhibitory control. Journal of the International Neuropsychological Society 13, 59-67.

Rosenzweig, M.R, Breedlove, S.M., \& Watson, N.V. (2007) Biological Psychology: An Introduction to Behavioral, Cognitive and Clinical Neuroscience (5th edition). Sunderland, MA: Sinauer Associates Inc.

Rydberg, S., Höhhielm, R. (1974) SPIQ Snabbt performancetest på intelligens. Stockholm: Skandinaviska testförlaget AB.

Salorio, F., Slomine, B., Guerguerian, A-M., Christensen, J. R., White, J. R. M., Natale, J. E.,..Gerring, J. P. (2008) Intensive care unit variables and outcome after pediatric traumatic brain injury: a retrospective study of survivors. Pediatric Critical Care Medicine 9, 47-53.

Saunders, L. L., Selassie, A. W., Hilla, E. G., Nicholas, J. S., Horner, M. D., Corrigan, J. D. \& Lackland, D. T. (2009) A population-based study of repetitive traumatic brain injury among persons with traumatic brain injury. Brain Injury 23, 866-872.

Savage, R. C., DePompei, R., Tyler, J. \& Lash, M. (2005) Paediatric traumatic brain injury: A review of pertinent issues. Pediatric Rehabilitation 8, 92-103.

Schneider, W. \& Pressley, M. (1997) Memory development between two and twenty, 2ed. New Jersey: Lawrence Erlbaum Associates, Publishers.

Sergui-Gomez, M. \& MacKenzie, E. J. (2003) Measuring the public health impact of injuries. Epidemiological Reviews 25, 3-19.

Slemmer, J. E., Matser, E. J. T., De Zeeuw, C. I. \& Weber, J. T. (2002) Repeated mild injury causes cumulative damage to hippocampal cells. Brain 125, 2699-2709.

Slomine, B., Gerring, J., Grados, M. A., Vasa, R., Brady, K. D., Cristensen, J. R. \& Denckla, M. B. (2002) Performance on measures of 'executive function' following pediatric traumatic brain injury. Brain Injury 16, 759-772. 
Slomine, B.S., Mc Carthy, M.L., Ding, R., Mac Kenzie, E.J., Jaffe, K.M., Aitken,,...Paidas, C.N. (2006) Health care utilization and needs after pediatric traumatic brain injury. Pediatrics 117, 663-674.

Smedler, A-C. \& Winiarski, J. (2008) Neuropsychological outcome in very young hematopoietic SCT recipients in relation to pretransplant conditioning. Bone Marrow Transplantation 42, 515-522.

Spreen O \& Strauss E. A (1998) Compendium of neuropsychological tests. 2ed. New York NY: Oxford University Press.

Stacin, T., Wade, S., Walz, N.C., Yeates, K.O. \& Taylor, G.H. (2008) Traumatic Brain Injuries in Early Childhood: Initial Impact on the Family. Journal of Development and Behavioural Pediatrics 29, 253-261.

Starmark, J-E., Stålhammar, D. \& Holmgren, E. (1988) The Reaction Level Scale (RLS 85) Manual and guidelines. Acta Neurochirurgica 91, 12-20.

Stern, Y. (2002). What is cognitive reserve? Theory and research application of the reserve concept. Journal of the International Neuropsychological Society 8, 448-460.

Tasker, R. C., Salmond, C. H., Westland, A. G., Pena, A., Gillard, J.H., Pena, A....Pickard, J. D. (2005) Head circumfence and Brain and Hippocampal volume after severe traumatic brain injury in childhood. Pediatric Research 58, 302-308.

Taylor G. (2004) Research on outcomes of pediatric traumatic brain injury: current advances and future directions. Developmental Neuropsychology $25,199-225$.

Taylor, G. \& Alden, J. (1997) Age-related differences in outcomes following childhood brain insults: An introduction and overview. Journal of the International Neuropsychological Society 3, 555-567.

Taylor, G.H., Swartwout, M.D., Yeates, K.O., Walz, N.C., Stacin, T. \& Wade, S.L. (2008) Traumatic brain injury in young children: Postacute effects on cognitive and school readiness skills. Journal of the International Neuropsychological Society 14, 734-745.

Taylor, H. G., Yeates, K. O., Wade, S. L., Drotar, D., Stacin, T. \& Minich, N. (2002) A prospective Study of Short- and Long-term Outcomes After Traumativ Brain Injury in Children: Behavior and Achievement. Neuropsychology 16, 15-27.

Teasdale, G. \& Jennett, B. (1974) Assessment of coma and impaired consciousness. A practical scale. The Lancet 13, 81-84.

Tepas, J.J., Leaphart, C.L., Pieper, P., Beaulieu, C.L., Spierre, L.R., Tuten, J.D. \& Celso, B.G. (2009) The effect of delay in rehabilitation on outcome of severe traumatic injury. Journal of Pediatric Surgery 44, 368 372 . 
Tombaugh, T., Kozak, J., Rees, L. (1999) Normative Data Stratified by Age and Education for Two Measures of Verbal Luency: FAS and Animal Naming. Archives of Clinical Neuropsychology 14, 167-177.

van't Hooft, I., Anderssson, K., Bergman, B., Sejersen, T., von Vendt, L. \& Bartfai, A. (2005) Beneficial effect from a cognitive training programme on children with acquired brain injuries demonstrated in a controlled study. Brain Injury 19, 511-518.

Van Heugten, C. M., Hendriksen, J., Rasquin, S., Dijcks, B., Jaeken, D. \& Vles, J.H.S. (2006) Long-term neuropsychological performance in a cohort of children and adolescents after severe paediatric traumatic brain injury. Brain Injury 20, 895-903.

Wechsler, D. (1977) Manual till WISC, 4th ed. Stockholm: Psykologiförlaget $\mathrm{AB}$.

Wechsler, D. (1996) Manual WAIS-R, 2th ed. Stockholm: Psykologiförlaget.

Wechsler, D. (1999) WISC III. Manual. Stockholm, Psykologiförlaget AB.

Vik, A., Kvistad, K.A., Skandsen, T. \& Ingebrigtsen, T. (2006) Diffuse axonal injury in traumatic brain injury. Tidsskrift for Den Norske Laegeforening 22, 2940-2944.

von Hofsten, C. (2004) An action perspective on motor development. Trends in Cognitive Sciences 8, 266-272.

Wahlström, M. R., Oliviecrona, M., Koskinen, L-O., Rydehag, B. \& Naredi, S. (2005) Severe traumatic brain injury in pediatric patients: treatment and outcome using an intracranial pressure targeted therapy - the Lund concept. Intensive Care Medicine 31, 832-839.

Walther, S. M., Jonasson, U. \& Gill, H. (2003) Comparison of the Glascow Coma Scale and the Reaction Level Scale for assessment of cerebral responsiveness in the critically ill. Intensive Care Medicine 29, 933-938.

Ward, H., Shum, D., Wallace, G. \& Boon, J. (2002) Pediatric Traumatic Brain Injury and Procedural memory. Journal of Clinical and Experimental Neuropsychology 24, 458-470.

Wilde, E. A., Bigler E. D., Hunter J.V., Fearing M. A., Scheibel R.

S.,Newsome M. R.,...Levin H. S. (2007) Hippocampus, amygdale and basal ganglia morphometrics in children after moderate-to-severe traumatic brain injury. Developmental Medicine and Child Neurology 49 294-299.

Yeates, K. O., Armstrong, K., Janusz, J., Taylor, G., Wade, S., Stancin, T., Drotar, D. (2005) Long-term attention problems in children with traumatic brain injury. The Journal of the American Academy of Child \& Adolescent Psychiatry 44, 574-584. 
Yeates, K. O. \& Enrile, B. G. (2005) Implicit and explicit memory in children with congenital and acquired brain disorder. Neuropsychology 19, 618-628.

Yeates, K., O., Swift, E., Taylor, .H G., Wade, S L., Drotar, D., Stacin, T. \& Minich, N. (2004) Short and Long-term social outcomes following pediatric traumatic brain injury. Journal of the International Neuropsychological Society 10, 412-416.

Yeates, K. O., Taylor, H. G., Wade, S. L., Drotar, D., Stacin, T. \& Minich, N. (2002) A Prospective Study of Short- and Long Term Neuropsychological Outcomes After Traumatic Brain Injury in Children. Neuropsychology 16, 514-523.

Yeudall, L. T., Fromm, D., Reddon, J. R. \& Stefanyk, W. O. (1986) Normative data stratified by age and sex for 12 neuropsychological tests. Journal of Clinical Psychology 42, 918-946.

Ylvisaker, M. (1998) Traumatic brain injury rehabilitation. Children and adolescents. Newton, MA: Butterworth-Heinemann.

Ylvisaker, M., Adelson, D., Braga, L. W., Burnett, S. M., Glang, A., Freeney, T.,...... Todis, B. (2005) Rehabilitation and ongoing support after pediatric TBI, Twenty years of progress. Journal of Head Trauma Rehabilitation 20, 95-109.

Ylvisaker, M., Turkstra, L., Coehlo, C., Yorkston, K., Kennedy, M, Moore, M.,....Avery, J. (2007) Behavioural interventions for children and adults with behaviour disorders after TBI: A systematic review of the evidence. Brain Injury 21, 769-805. 